\title{
Reconstruction of solar total irradiance since 1700 from the surface magnetic flux
}

\author{
N. A. Krivova, L. Balmaceda, and S. K. Solanki \\ Max-Planck-Institut für Sonnensystemforschung, Max-Planck-Str. 2, 37191 Katlenburg-Lindau, Germany \\ e-mail: natalie@mps.mpg.de
}

Received 9 November 2006 / Accepted 23 February 2007

\section{ABSTRACT}

\begin{abstract}
Context. Total solar irradiance changes by about $0.1 \%$ between solar activity maximum and minimum. Accurate measurements of this quantity are only available since 1978 and do not provide information on longer-term secular trends.

Aims. In order to reliably evaluate the Sun's role in recent global climate change, longer time series are, however, needed. They can only be assessed with the help of suitable models.

Methods. The total solar irradiance is reconstructed from the end of the Maunder minimum to the present based on variations of the surface distribution of the solar magnetic field. The latter is calculated from the historical record of the sunspot number using a simple but consistent physical model.

Results. Our model successfully reproduces three independent data sets: total solar irradiance measurements available since 1978, total photospheric magnetic flux since 1974 and the open magnetic flux since 1868 empirically reconstructed using the geomagnetic $a a$-index. The model predicts an increase in the solar total irradiance since the Maunder minimum of $1.3_{-0.4}^{+0.2} \mathrm{Wm}^{-2}$.
\end{abstract}

Key words. Sun: activity - Sun: faculae, plages - Sun: magnetic fields - solar-terrestrial relations - sunspots

\section{Introduction}

Variations in solar total (i.e. integrated over all wavelengths) and spectral irradiance have been measured by a number of partly overlapping space experiments since 1978 (e.g., Willson \& Hudson 1988, 1991; Brueckner et al. 1993; Woods et al. 1996; Floyd et al. 2003; Fröhlich 2003, and references therein). There is strong interest in this quantity due to its potential influence on global climate. Many investigations have considered whether and how the observed variations in solar irradiance influence the Earth's climate (e.g., Haigh 1996, 1999; Kodera \& Kuroda 2002; Coughlin \& Tung 2004; Arnold 2005; Kuroda \& Kodera 2005; Egorova et al. 2005; Langematz et al. 2005; Sinnhuber et al. 2005; Dameris et al. 2006). Variations of solar irradiance on longer time scales, which have not been directly measured, are possibly of even greater importance for the global climate change (e.g., Eddy 1976; Reid 1987; Bond et al. 2001; Haigh 2001; Shindell 2001; Rind 2002; Stott et al. 2003), since the coupled system of the Earth's atmosphere and oceans reacts rather slowly to the varying solar signal. Hence understanding the mechanisms of solar impact on the Earth's environment is plagued by the inadequate length of the directly measured time series of solar irradiance. Only a reconstruction of solar irradiance for the pre-satellite period with the help of models can aid in gaining further insight into the nature of this influence.

A number of long-term (up to 300-400 years) reconstructions, both purely empirical and more physics-based, have been produced in the last two decades (for more details and references see the overviews by, e.g., Solanki \& Krivova 2004; Lockwood 2005). Such reconstructions are usually split into two parts: reconstruction of a cyclic component and a secular variation. The reconstruction of the cyclic component is relatively straightforward from various historical proxy records of solar magnetic activity, such as the (Group and Zurich) sunspot number, sunspot areas, white-light facular areas, Ca II plage areas, etc. (e.g., Foukal \& Lean 1990; Hoyt \& Schatten 1993; Lean et al. 1995, 2001; Solanki \& Fligge 1998, 1999; Fligge \& Solanki 2000; Preminger \& Walton 2005).

The magnitude of the secular change in the irradiance is far more speculative. Evidence for such a long-term trend was initially provided by stellar data. Baliunas \& Jastrow (1990) found that the distribution of $\mathrm{Ca}$ II $\mathrm{H}$ and $\mathrm{K}$ emission in field stars fell into two groups. They argued that stars with the lowest Ca II brightness do not show any cyclic modulations and are thus in a state similar to the Maunder minimum. On the other hand, the Sun's Ca II level at recent activity minima remained markedly higher than that of most other late-type stars in the Baliunas \& Jastrow sample (White et al. 1992). This could mean that the Sun during the Maunder minimum was much more quiet than during current minima, e.g. due to disappearance of the network. Assuming that the relationship between solar Ca II brightness and irradiance did not change, estimates of the irradiance increase since that time encompassed the range between 2 and $16 \mathrm{Wm}^{-2}$ (Lean et al. 1992; Zhang et al. 1994; Mendoza 1997).

The stellar evidence is indirect and based on a number of assumptions. For example, extrapolation of the relation between $\mathrm{Ca}$ II flux and the irradiance measured for recent cycles to lower levels of activity is based on the assumption that this relationship is the same for the quiet Sun and active regions (which differ, e.g., in the absence and presence of sunspots). Also, recent measurements of carefully selected samples of stars suggest that many of the "Maunder minimum" stars in the Baliunas \& Jastrow sample had, in fact, evolved from the main sequence (Wright 2004). In addition, stars in a Maunder minimum-like state do not always exhibit $\mathrm{Ca}$ II emission brightness below that of solar minimum (Hall \& Lockwood 2004). 
The problems faced by the stellar approach have recently led to the development of alternative methods. Thus, assuming that faculae are located near sunspots, Foster (2004), cf. Lockwood (2005), recontsructed the butterfly diagram for faculae using the historical record of sunspot areas and positions. He then employed averaged functions for the centre-to-limb variations of the facular contrasts derived by Ortiz et al. (2002), in order to model facular brightening. Analysing the distribution of the magnetic field in the recent period and assuming that the Sun was magnetically quiet during the Maunder minimum Foster (2004) set an upper limit of $\approx 1.7 \mathrm{Wm}^{-2}$ on the secular variation since that time. This limit increases to approx $2.1 \mathrm{Wm}^{-2}$ if the weak magnetic flux escaping registration in the Michelson Doppler Imager (MDI) magnetograms due to insufficient spatial resolution (Krivova \& Solanki 2004) is taken into account. Additional uncertainty in this model is introduced by the fact that the contrasts derived at a single wavelength are used instead of more realistic facular models.

A yet lower value of about $1 \mathrm{Wm}^{-2}$ for the increase in the cycle-averaged total solar irradiance (TSI) since the beginning of the 18th century was published by Wang et al. (2005), who used a flux transport model to simulate the evolution of the solar magnetic flux and then the TSI. Solanki et al. $(2000,2002)$ presented a simple physical model that allows a reconstruction of the solar total and open magnetic flux from the sunspot number and provides an explanation for the origin of the secular variation displayed by the open flux. Here we use this model to reconstruct the solar total irradiance variations back to 1700 using the Zurich sunspot number and to 1610 using the Group sunspot number. In our approach we follow the successful irradiance modelling by, e.g., Wenzler et al. (2006) as closely as possible and extend it to longer times. We also constrain our reconstructions by requiring them to reproduce the available time series of total solar irradiance as well as total and open magnetic flux. We describe our approach in Sect. 2, present and discuss the results in Sect. 3 and sum up our conclusions in Sect. 4.

\section{Approach}

Our model describes irradiance changes on time scales of days to centuries that are due to the evolution of the solar surface magnetic flux. Models based on this assumption explain about $90 \%$ of all irradiance changes observed on time scales of a day to decades (Krivova et al. 2003; Wenzler et al. 2004, 2005, 2006). Of course, we cannot rule out that other effects also influence irradiance on longer time scales (e.g., changes in convection or thermal shadows from subsurface flux, e.g., Parker 1987, 1995), but since there is no observational evidence for that, we here ignore all other possible influences.

The success of the model by Krivova et al. (2003) and Wenzler et al. $(2005,2006)$ implies that if the evolution of the magnetic flux is known for each photospheric component (sunspots, faculae and the network), then it is also possible to calculate irradiance variations. Unfortunately, measurements of the magnetic flux are not available prior to the second half of the 20 th century, so that it has to be computed based on some proxy. A model that allows a reconstruction of the solar magnetic flux from the sunspot number has been worked out by Solanki et al. (2002). We outline the idea of this model in Sect. 2.1, describe how we use it for the irradiance reconstructions in Sect. 2.2 and review the model parameters and the available observational data that need to be reproduced by the model in Sect. 2.3.

\subsection{Photospheric magnetic flux}

For simplicity, we divide the continuous spectrum of sizes of magnetic structures on the solar surface into active and ephemeral regions. Active regions (AR) are larger bipolar structures emerging in the activity belts at latitudes below approximately $\pm 30^{\circ}$ and living up to several weeks. They generally contain one or more sunspots. The flux emergence rate in active regions can be estimated from the historical record of the sunspot number.

Ephemeral regions (ER) emerge at all latitudes in the form of smaller, short-lived bipoles. They do not contain sunspots. Due to their high emergence rate (Harvey 1993; Hagenaar 2001) their contribution to the total photospheric magnetic flux is significant. ER evolve over a cycle which is extended with respect to the sunspot cycle (e.g., Harvey 1992, 1994a). In order to describe their evolution in time, we assume that the length and the amplitude of the ER cycle are related to the properties of the corresponding sunspot cycle, i.e. we assume that they are produced by the main dynamo, just like the active regions.

Most of the emerged photospheric flux forms closed loops until it decays due to cancellation with flux of opposite polarity. A small part of it is, however, dragged outward by the coronal gas and reaches far into the heliosphere. This is called the open magnetic flux. Both active and ephemeral regions contribute to the open flux, which can survive on the solar surface for several years, since it is located in often large regions with a dominant magnetic polarity (e.g., at the poles).

A set of three coupled ordinary differential equations has been shown to describe the evolution of the three flux components (Solanki et al. 2002):

$$
\begin{aligned}
& \frac{\mathrm{d} \phi_{\mathrm{act}}}{\mathrm{d} t}=\varepsilon_{\mathrm{act}}(t)-\frac{\phi_{\mathrm{act}}}{\tau_{\mathrm{act}}}-\frac{\phi_{\mathrm{act}}}{\tau_{\mathrm{ta}}} \\
& \frac{\mathrm{d} \phi_{\mathrm{eph}}}{\mathrm{d} t}=\varepsilon_{\mathrm{eph}}(t)-\frac{\phi_{\mathrm{eph}}}{\tau_{\mathrm{eph}}}-\frac{\phi_{\mathrm{eph}}}{\tau_{\mathrm{te}}} \\
& \frac{\mathrm{d} \phi_{\mathrm{open}}}{\mathrm{d} t}=\frac{\phi_{\mathrm{act}}}{\tau_{\mathrm{ta}}}+\frac{\phi_{\mathrm{eph}}}{\tau_{\mathrm{te}}}-\frac{\phi_{\mathrm{open}}}{\tau_{\mathrm{open}}}
\end{aligned}
$$

Here $\phi_{\text {act }}, \phi_{\text {eph }}$ and $\phi_{\text {open }}$ refer to the magnetic flux of AR, ER and open components, respectively, and their sum represents the total photospheric magnetic flux, $\phi_{\mathrm{tot}}$ :

$\phi_{\text {tot }}=\phi_{\text {act }}+\phi_{\text {eph }}+\phi_{\text {open }}$.

The main ingredients entering the model are the flux emergence rates, $\varepsilon_{\text {act }}$ and $\varepsilon_{\text {eph }}$, of AR and ER, respectively, the decay time scales for the three flux components, $\tau_{\text {act }}, \tau_{\text {eph }}, \tau_{\text {open }}$, and the time scales for flux transfer from active and ephemeral regions to the open flux, $\tau_{\text {ta }}$ and $\tau_{\text {te }}$, respectively. Equations (1)-(3) are solved following Solanki et al. (2002).

The flux emergence rate in active regions, $\varepsilon_{\text {act }}$, is chosen to vary with time, $t$, linearly with the sunspot number, $R$, and is scaled according to the observations of Schrijver \& Harvey (1994) for cycle 21:

$\varepsilon_{\mathrm{act}}(t)=\varepsilon_{\mathrm{act}, \max , 21} \frac{R(t)}{R_{\max , 21}}$,

with $\varepsilon_{\mathrm{act}, \max , 21}=2.3 \times 10^{24} \mathrm{Mx} \mathrm{yr}^{-1}$ and $R_{\max , 21}=150$.

Following Harvey (1993) we prescribe that $\varepsilon_{\text {eph }}(t)$ also varies cyclically, with the amplitude and the length of this cycle being related to the properties of the corresponding AR cycle:

$\varepsilon_{\mathrm{eph}, i}(t)=X \varepsilon_{\mathrm{act}, \mathrm{max}, \mathrm{i}} \sin ^{2}\left(t^{\prime}\right)$, 
with

$t^{\prime}=\left(t-t_{i}+\tau_{x}\right) \frac{\pi}{2 \tau_{x}+\tau_{i}}$.

Here $\tau_{i}=t_{i+1}-t_{i}$ is the length of the $i$ th sunspot cycle $\left(t_{i}\right.$ and $t_{i+1}$ refer to the times of the adjacent sunspot minima) and $\tau_{i}+2 \tau_{x}$ is the length of the corresponding ER cycle, which is, in line with observations (Harvey 1993), stretched by the interval $\tau_{x}$ in each direction with respect to the sunspot cycle. The value of $\tau_{x}$ depends on the length of the sunspot cycle: $\tau_{x}=\tau_{i}-c_{x}$, with $c_{x}$ lying in the range 5-9 years.

The time-independent amplitude factor $X$ in Eq. (6) can be estimated in the following manner:

$X=\frac{\varepsilon_{\text {eph,max }}}{\varepsilon_{\text {eph,min }}} \frac{\varepsilon_{\text {eph,min }}}{\varepsilon_{\text {act,max }}}$.

Here $\varepsilon_{\text {eph,min }}=1.8 \times 10^{26} \mathrm{Mx} \mathrm{yr}^{-1}\left(\right.$ Hagenaar 2001),$\varepsilon_{\text {act, } \max }=$ $2.3 \times 10^{24} \mathrm{Mx} \mathrm{yr}^{-1}$ (Schrijver \& Harvey 1994), and values of $\varepsilon_{\text {eph,max }} / \varepsilon_{\text {eph,min }}$ found in the literature lie in the range 1-2 (Harvey 1993, 1994b; Hagenaar et al. 2003). These values result in $X \approx 80-160$.

The decay time of the AR flux can be assessed by assuming a balance between flux emergence and decay: $\tau_{\text {act }}=\phi_{\text {act }} / \varepsilon_{\text {act }}$. Employing the values $\phi_{\text {act, max }} \approx 6 \times 10^{23} \mathrm{Mx}$ and $\varepsilon_{\text {act, } \max }=2.3 \times$ $10^{24} \mathrm{Mx} \mathrm{yr}^{-1}$ for the maximum of cycle 21 , which is the best estimate obtained from the plots in Harvey (1994b); Schrijver \& Harvey (1994), we arrive at an estimate of $\tau_{\text {act }} \approx 0.25 \mathrm{yr}$. For the decay time of the ER flux we take the value of $\tau_{\mathrm{eph}}=14 \mathrm{~h}$ as found by Hagenaar (2001).

The transfer times from AR and ER flux to the open flux, $\tau_{\text {ta }}$ and $\tau_{\text {te }}$, are considerably longer ( $\gtrsim 10 \mathrm{yr}$ ) than the corresponding decay times and are not expected to influence the evolution of $\phi_{\text {act }}$ and $\phi_{\text {eph }}$ significantly. We varied $\tau_{\text {ta }}$ within the range 10 to 14 years, and take $\tau_{\text {te }}=6 \tau_{\text {ta }}$ according to Harvey (1994b). These parameters only affect the open flux together with the decay time of the open flux, $\tau_{\text {open }}$, lying in the range 2-6 years.

\subsection{Solar irradiance}

Our approach to the irradiance reconstruction is similar in concept to that of the SATIRE models (Spectral And Total Iradiance REconstructions; see Solanki et al. 2005; Krivova \& Solanki 2005 ) used to calculate solar irradiance on time scales of days to the solar cycle (Krivova et al. 2003; Wenzler et al. 2004, 2005, 2006). We divide the solar photosphere into 5 components: quiet Sun (index q from here on), sunspot umbrae (u) and penumbrae (p), faculae (f) and the network (n). The time-independent brightness of each component $F_{\mathrm{q}, \mathrm{u}, \mathrm{p}, \mathrm{f}, \mathrm{n}}(\lambda)$, where $\lambda$ is the wavelength, is calculated using the ATLAS9 code of Kurucz from plane-parallel model atmospheres (for details, see Unruh et al. 1999). Note that we use the same model for faculae and the network: $F_{\mathrm{f}}=F_{\mathrm{n}}$. The variation of the solar irradiance with time is described by the corresponding filling factors, $\alpha_{\mathrm{q}, \mathrm{u}, \mathrm{p}, \mathrm{f}, \mathrm{n}}(t)$, which define the fraction of the solar surface covered by each component at a given time. Since magnetograms, from which the $\alpha$ values have in the past mainly been determined, are not available for most of the time since the Maunder minimum, we need to consider other sources for $\alpha$.

Evaluation of filling factors for sunspots is straightforward. In the period since 1874 the sunspot areas were directly recorded at a number of observatories. The longest and most complete time series is that of the Royal Greenwich Observatory (1874-1976), which is used as a basis for our

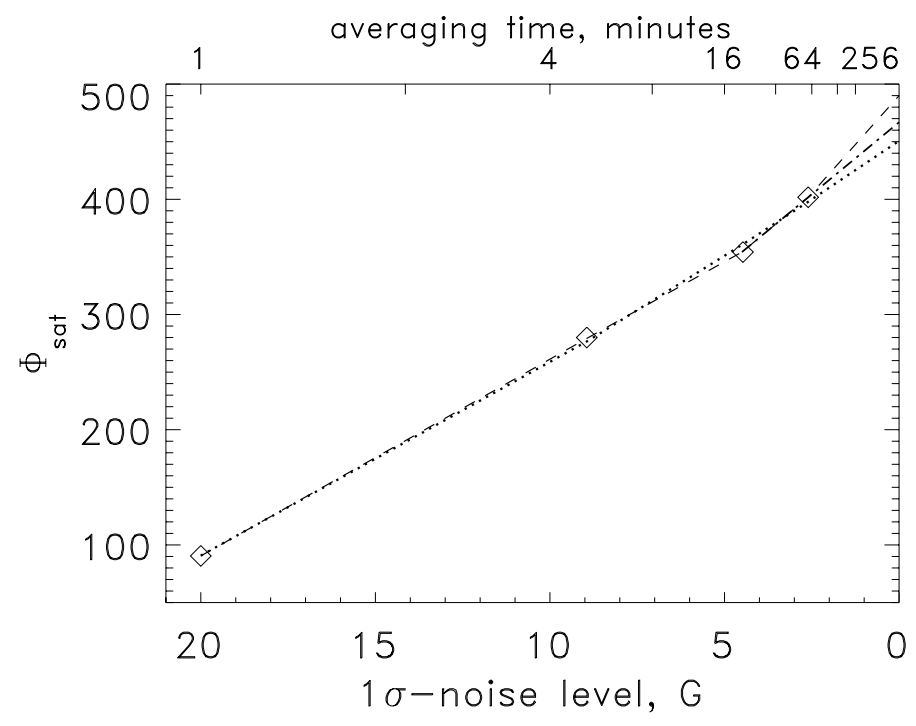

Fig. 1. Dependence of the saturation flux, $\phi_{\text {sat }}$, on the noise level of the employed magnetograms. Values of $\phi_{\text {sat }}$ obtained with the original 1minute magnetograms and their 5-, 20- and 59-min averages (having the $1 \sigma$ noise level of approximately $20 \mathrm{G}, 8.9 \mathrm{G}, 4.5 \mathrm{G}$ and $2.6 \mathrm{G}$, respectively) are marked by diamonds. The lines show three different fits to these points and their extrapolation to the zero noise level: a linear fit (dot-dashed line) based on 2 points with the lowest noise $(\sigma=2.6 \mathrm{G}$ and $4.5 \mathrm{G}$ ) gives an estimate of $\phi_{\text {sat }}(0)=467 \mathrm{G}$ for $\sigma=0 \mathrm{G}$, whereas quadratic (dotted) and cubic (dashed) fits to all points yield $\phi_{\text {sat }}(0)=$ $451 \mathrm{G}$ and $490 \mathrm{G}$, respectively.

compillation. For the period 1977-1985, we use the data obtained in the former USSR and for the most recent period (since 1986) those compiled by the USAF (US Air Force) network including the data from Mt. Wilson Observatory. The remaining gaps are filled in with the data from Rome, Yunnan and Catania observatories. Since records by different stations show systematic differences, they have been carefully analysed and cross-calibrated (see Balmaceda et al. 2005, for further details). Before 1874 sunspot area measurements are not available, but can easily be extrapolated through a correlation analysis with the sunspot number. We alternately use the Zurich sunspot number, $R_{\mathrm{Z}}$ (Waldmeier 1961), and the Group sunspot number, $R_{\mathrm{G}}$ (Hoyt \& Schatten 1993), provided by the National Geophysical Data Center (NGDC, http:// www.ngdc.noaa.gov/stp/SOLAR/solar.html). Only yearly values of $R_{\mathrm{Z}}$ are available for 1700-1818, monthly records are accessible for 1819-1848, whereas daily numbers have been stored since 1849 . We use a fixed ratio between umbral and penumbral areas, $\alpha_{\mathrm{u}} /\left(\alpha_{\mathrm{u}}+\alpha_{\mathrm{p}}\right)=0.2$ as obtained by Wenzler (2005) for recent cycles and in agreement with other estimates reported in the literature (for an overview see Solanki 2003).

The filling factors of other photospheric components are obtained from the magnetic flux calculated as outlined in Sect. 2.1. The sum of the ER and open fluxes describes the evolution of the network: $\phi_{\mathrm{n}}=\phi_{\mathrm{eph}}+\phi_{\text {open }}$, whereas $\phi_{\text {act }}$ represents the variation of the magnetic flux in sunspots and faculae. The magnetic flux in sunspots (the sum of the umbral, $\phi_{\mathrm{u}}$, and penumbral, $\phi_{\mathrm{p}}$, components) can be calculated from the sunspot areas:

$\phi_{\mathrm{u}}+\phi_{\mathrm{p}}=\alpha_{\mathrm{u}}\left\langle B_{z, \mathrm{u}}\right\rangle+\alpha_{\mathrm{p}}\left\langle B_{z, \mathrm{p}}\right\rangle$,

where the area-averaged vertical components of the magnetic flux strength in umbrae, $\left\langle B_{z, \mathrm{u}}\right\rangle$, and penumbrae, $\left\langle B_{z, \mathrm{p}}\right\rangle$, are about $1800 \mathrm{G}$ and $550 \mathrm{G}$, respectively, according to the measurements 
Table 1. Key features of the models providing best fits to the 3 considered data sets. (1) Main parameters (i.e. those that are also varied in the standard model). These include $c_{x}=\tau_{i}-\tau_{x}$ (where $\tau_{i}$ is the length of the AR cycle and $\tau_{i}+2 \tau_{x}$ is the length of the corresponding ER cycle), the amplitude factor $X$ (see Eqs. (6) and (8)), the flux transfer time from AR to the open flux, $\tau_{\text {ta }}$, and the decay time for the open flux, $\tau_{\text {open. }}$. In models "antiphase (2)" and "const. $\phi_{\mathrm{eph}}(2)$ ", an optional parameter is the saturation flux in faculae, $\phi_{\mathrm{sat}, \mathrm{f}}$, which is otherwise fixed to $300 \mathrm{G}$. Also listed are (2) the corresponding values of the variance (i.e. $\chi^{2}$ normalised by the number of points) for the TSI, $\sigma_{\mathrm{TSI}}^{2}$, total magnetic flux, $\sigma_{\mathrm{TMF}}^{2}$ and the open flux, $\sigma_{\mathrm{open}}^{2}$, their mean, $\sigma^{2}=\left(\sigma_{\mathrm{TSI}}^{2}+\sigma_{\mathrm{TMF}}^{2}+\sigma_{\mathrm{open}}^{2}\right) / 3$, the correlation coefficient, $R_{\mathrm{c}, \mathrm{TSI}}$, between the modelled and measured TSI and (3) the estimated increase in the TSI since 1700. In the last row, ranges within which all parameters have been varied are listed.

\begin{tabular}{|c|c|c|c|c|c|c|c|c|c|c|c|c|}
\hline \multirow[b]{2}{*}{ Model } & \multirow[b]{2}{*}{ Sect. } & \multicolumn{5}{|c|}{$\begin{array}{c}(1) \\
\text { Parameters }\end{array}$} & \multicolumn{5}{|c|}{$\begin{array}{l}\text { (2) } \\
\text { Fit quality }\end{array}$} & \multirow{2}{*}{$\begin{array}{c}(3) \\
\text { Predicted } \\
\Delta I, \mathrm{Wm}^{-2} \\
\text { since } 1700\end{array}$} \\
\hline & & $\begin{array}{l}c_{x}, \\
\mathrm{yr}\end{array}$ & $X$ & $\begin{array}{c}\tau_{\mathrm{ta}}, \\
\mathrm{yr}\end{array}$ & $\begin{array}{c}\tau_{\text {open }}, \\
\mathrm{yr}\end{array}$ & $\begin{array}{c}\phi_{\text {sat,f }}, \\
\mathrm{G}\end{array}$ & $\sigma_{\text {TSI }}^{2}$ & $\sigma_{\mathrm{TMF}}^{2}$ & $\sigma_{\text {open }}^{2}$ & $\sigma^{2}$ & $R_{\mathrm{c}, \mathrm{TSI}}$ & \\
\hline standard $R_{\mathrm{Z}}$ & 3.1 & 7.6 & 117 & 13.5 & 3.5 & $300^{\star}$ & 0.048 & 0.0062 & 0.041 & 0.032 & 0.78 & $\approx 1.3$ \\
\hline antiphase $^{+}$(1) & 3.2 & 5.0 & 80 & 10.0 & 2.6 & $300^{\star}$ & 0.113 & 0.0071 & 0.041 & 0.054 & 0.64 & \\
\hline antiphase $^{+}(2)$ & 3.2 & 7.4 & 80 & 13.9 & 3.9 & 168 & 0.055 & 0.0086 & 0.036 & 0.033 & 0.77 & $\approx 1.5$ \\
\hline const. $\phi_{\text {eph }}(1)$ & 3.3 & $-^{\#}$ & ${ }^{\#}$ & 10.0 & 2.5 & $300^{\star}$ & 0.116 & 0.0072 & 0.038 & 0.054 & 0.63 & \\
\hline const. $\phi_{\mathrm{eph}}(2)$ & 3.3 & $-^{\#}$ & ${ }^{*}$ & 13.5 & 3.6 & 182 & 0.049 & 0.0074 & 0.035 & 0.031 & 0.77 & $\approx 0.9$ \\
\hline standard $R_{\mathrm{G}}$ & 3.4 & 7.6 & 117 & 13.5 & 3.5 & $300^{\star}$ & 0.049 & 0.0062 & 0.035 & 0.030 & 0.75 & $\approx 1.3$ \\
\hline ranges & 2 & $5-9$ & $80-160$ & $10-14$ & $2-6$ & $150-400$ & & & & & & \\
\hline
\end{tabular}

* this parameter has been deduced independently of the current modelling and its value was not varied in order to fit the data.

${ }^{+}$in the antiphase model, the shift between the emergence rate of AR and ER was varied between 2 and 8 years (ER preceding AR), so that it becomes an additional free parameter. For the best-fit models presented here, the shift is 5 years.

\# in the const. $\phi_{\mathrm{eph}}$ model, $c_{x}$ and $X$ are replaced by one free parameter: $\phi_{\mathrm{eph}}=3.0 \times 10^{15} \mathrm{~Wb}$ for model (1) and $\phi_{\mathrm{eph}}=2.8 \times 10^{15} \mathrm{~Wb}$ for model (2).

of Keppens \& Martínez Pillet (1996). Subtraction of $\phi_{\mathrm{u}}$ and $\phi_{\mathrm{p}}$ from $\phi_{\text {act }}$ gives the evolution of the facular magnetic flux, $\phi_{\mathrm{f}}$.

Next, we convert the network, $\phi_{\mathrm{n}}$, and facular, $\phi_{\mathrm{f}}$, magnetic fluxes into corresponding filling factors, $\alpha_{\mathrm{n}}$ and $\alpha_{\mathrm{f}}$. For this, we use the same conversion scheme as in previous applications of the SATIRE models (Krivova et al. 2003; Wenzler et al. 2004, 2005, 2006): $\alpha_{\mathrm{n}}$ and $\alpha_{\mathrm{f}}$ are proportional to $\phi_{\mathrm{n}}$ and $\phi_{\mathrm{f}}$, respectively, until a saturation limit $\phi_{\text {sat }}$ is reached where $\alpha=1$ (see previous papers for more details). Above $\phi_{\text {sat }}$ they remain constant. $\phi_{\text {sat }}$ is a free parameter of the SATIRE models, which depends on the resolution and the noise level of the employed magnetic field maps. The smaller the fraction of the true magnetic flux visible in the magnetograms, the smaller the $\phi_{\text {sat }}$ needs to be.

In order to reproduce solar irradiance variations in cycle 23 on the basis of MDI magnetograms, Krivova et al. (2003) found $\phi_{\text {sat }}=280 \mathrm{G}$ (no distinction was made between faculae and the network). For a reconstruction of solar irradiance in cycles 2123 based on National Solar Observatory Kitt Peak (NSO KP) data, Wenzler et al. $(2005,2006)$ found $\phi_{\text {sat }}=310-320 \mathrm{G}$ to be more adequate. The larger $\phi_{\text {sat }}$ is consistent with the fact that KP magnetograms have a lower noise level than those from the MDI. Since magnetic field strengths in faculae are higher than the noise level of either of these 2 sets of images, we do not

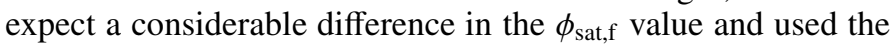
value of $300 \mathrm{G}$ as our primary estimate. In some extreme cases (see Sects. 3.2 and 3.3), however, we allowed $\phi_{\text {sat, f }}$ to vary.

Faculae mainly appear in the so-called activity belts (i.e. at latitutes between approximately $\pm(5-15)$ and $\pm(40-45)$ degrees), and their distribution therefore peaks at $\mu$-values above 0.9 (see, e.g., Fig. 11b in Wenzler et al. 2006). However, faculae become fainter when they cross the disc centre (e.g., Ortiz et al. 2002). By assuming a homogeneous distribution of faculae over the solar surface, we overestimate their contribution to the brightening of the Sun, since the ratio of low- $\mu$ to large- $\mu$ area is smaller for activity belts than for the whole disc. We take this into account by weighting facular contributions, with weights proportional to the fraction of the solar disc occupied by each $\mu$-bin within activity belts and 0 outside of these.
The weakest network elements are lost within the noise level and, since they are often present as mixed polarity features, they are also lost due to apparent cancellation of polarities caused by insufficient spatial resolution of the MDI or KP magnetograms (Krivova \& Solanki 2004). Consequently, in case of noise-free high resolution data, their saturation flux could lie significantly higher than $300 \mathrm{G}$ deduced from MDI and KP magnetograms. In order to estimate $\phi_{\text {sat,n }}$ for such ideal "noise-free" data, we utilised MDI magnetograms with different noise levels to recontsruct solar irradiance on 70 days between the minimum of cycle 23 in 1996 and its maximum in 2000 following Krivova et al. (2003). For this, we consecutively averaged MDI magnetograms over 5, 20 or 59 min (which reduced the $1 \sigma$ noise level down to approximately $8.9 \mathrm{G}, 4.5 \mathrm{G}$ and $2.6 \mathrm{G}$, respectively) and employed these together with the original 1-min data (having the $1 \sigma$ noise level of about $20 \mathrm{G}$ ) for the reconstruction. In each case, the value of $\phi_{\text {sat }}$ was found from a comparison with the irradiance variations measured by the VIRGO instrument on SoHO (Fröhlich \& Finsterle 2001). The dependence of the $\phi_{\text {sat }}$ on the noise level of the employed magnetograms is shown in Fig. 1. An extrapolation to the 0-noise level (see Fig. 1) suggests a value of $\phi_{\text {sat, } n}$ of around $500 \mathrm{G}$, which we use in this work.

The magnetic fluxes reconstruted with the model described in Sect. 2.1 are integrals over the solar disc. Thus the filling factors obtained using $\phi_{\mathrm{f}}$ and $\phi_{\mathrm{n}}$ are averages over the solar disc:

$\left\langle\alpha_{\mathrm{f}, \mathrm{n}}\right\rangle=\max \left[\frac{\left\langle\phi_{\mathrm{f}, \mathrm{n}}\right\rangle}{\phi_{\mathrm{sat}}}, 1\right]$,

where $\left\langle\phi_{\mathrm{f}, \mathrm{n}}\right\rangle$ refer to the magnetic fluxes from faculae or the network averaged over the solar disc. These filling factors can be somewhat higher than the disc-averaged filling factors obtained with spatially resolved data as used by Krivova et al. (2003) and Wenzler et al. (2004, 2005, 2006), which are

$\left\langle\alpha_{\mathrm{f}, \mathrm{n}}^{\mathrm{resolved}}\right\rangle=\frac{1}{N} \sum_{i=0, N} \alpha_{\mathrm{f}, \mathrm{n}}^{i}$. 

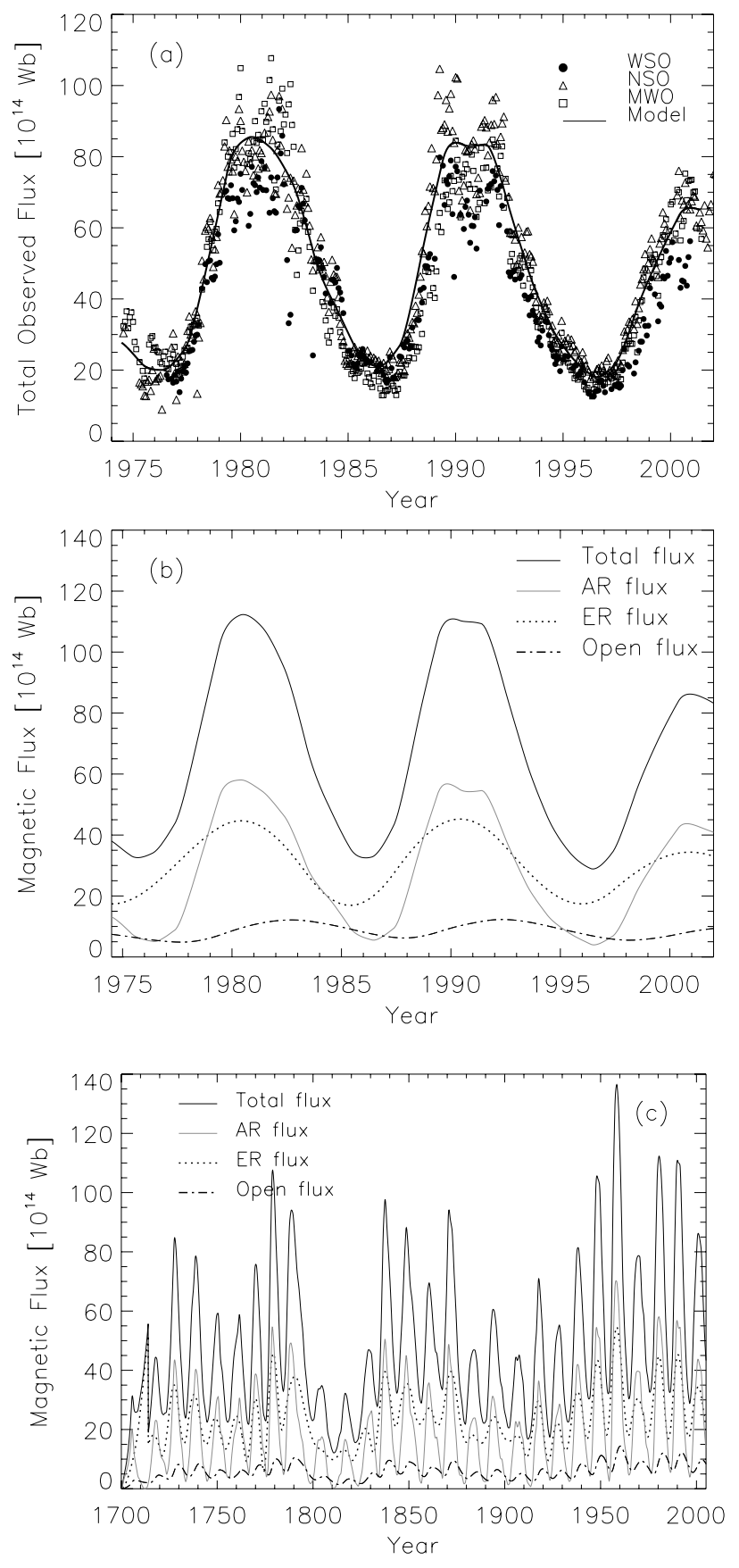

Fig. 2. a) Measured (symbols) and modelled (standard model; solid line) total magnetic flux between 1974 and 2002. Each data point is an integral over a synoptic chart for one Carrington rotation. Different symbols are used for different data sets: filled circles represent the WSO data, open triangles the KP NSO data and open squares the MWO measurements (cf. Arge et al. 2002; Wenzler et al. 2006). For the modelled flux the value $\phi_{\text {act }}+0.4 \phi_{\text {eph }}+\phi_{\text {open }}$ is given (see Sect. 2.3). b) and c) Reconstructed magnetic flux in AR (grey solid curve), ER (dotted), as well as the open (dot-dashed) and total (black solid) flux since 1974 and 1700 , respectively.

Here $\alpha_{\mathrm{f}, \mathrm{n}}^{i}$ is the filling factor for each individual pixel of the employed magnetogram:

$\alpha_{\mathrm{f}, \mathrm{n}}^{i}=\max \left[\frac{\phi_{\mathrm{f}, \mathrm{n}}^{i}}{\phi_{\mathrm{sat}}}, 1\right]$,

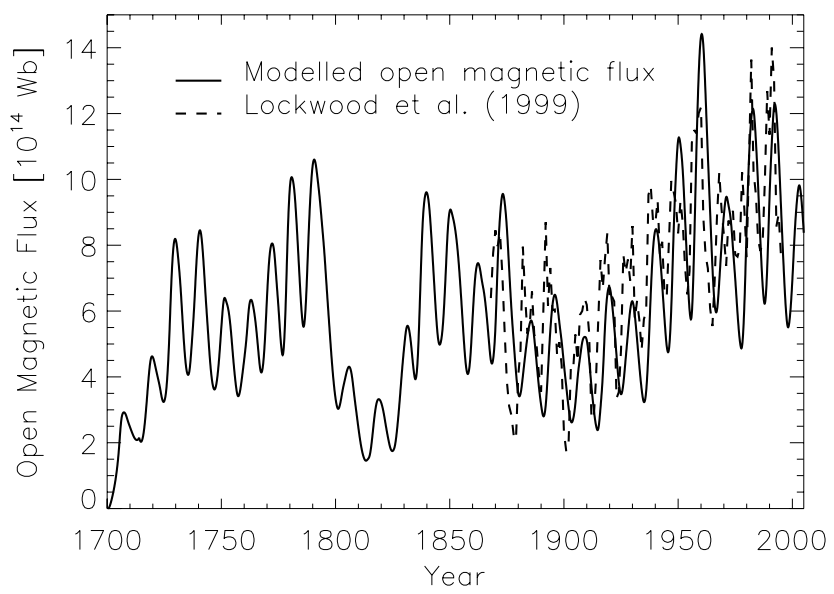

Fig. 3. Evolution of the open flux since 1700: calculated with the standard model (solid curve) and reconstructed by Lockwood et al. (1999) from the geomagnetic $a a$-index (dashed).

with $\phi_{\mathrm{f}, \mathrm{n}}^{i}$ being the magnetic flux for the same pixel. Note that $\left\langle\phi_{\mathrm{f}, \mathrm{n}}\right\rangle$, being an average over the disc, is always smaller than $\phi_{\mathrm{sat}}$, so that it never saturates. $\phi_{\mathrm{f}, \mathrm{n}}^{i}$ on the other hand is considerably larger than $\phi_{\mathrm{sat}}$ for at least some pixels. At these pixels $\alpha_{\mathrm{f}, \mathrm{n}}^{i}$ is saturated to 1 , so that they give a disproportionately smaller contribution compared to simply adding up all the $\phi_{\mathrm{f}, \mathrm{n}}^{i} / \phi_{\mathrm{sat}}$ values. Thus $\left\langle\alpha_{\mathrm{f}, \mathrm{n}}\right\rangle$ is somewhat larger than $1 / N \sum_{i=0, N} \alpha_{\mathrm{f}, \mathrm{n}}^{i}$. However, as can be seen from the histograms of the magnetic flux distribution in magnetograms (see, e.g., Fig. 11a of Wenzler et al. 2006), this distribution is exponential with the maximum reached for weakest fluxes. Thus the number of pixels falls off quickly with the increasing magnetic signal, such that only a few percent and even less than $1 \%$ of all pixels have magnetic fluxes higher than $300 \mathrm{G}$ and $500 \mathrm{G}$, respectively. Again, due to the exponential distribution, the dominant part of these "saturated" pixels lie immediately above the corresponding thresholds, and thus the errors introduced by this effect into the values of $\left\langle\alpha_{\mathrm{f}, \mathrm{n}}\right\rangle$ are yet smaller.

Finally, the area not covered by sunspots, faculae or the network is assumed to be free of magnetic field and is considered to be quiet Sun. The corresponding filling factor is $\alpha_{\mathrm{q}}=$ $1-\alpha_{\mathrm{u}}-\alpha_{\mathrm{p}}-\alpha_{\mathrm{f}}-\alpha_{\mathrm{n}}$, and the final formula for the solar radiative flux at a given wavelengths, $\lambda$, is:

$$
\begin{aligned}
F(\lambda, t)= & \alpha_{\mathrm{q}}(t) F_{\mathrm{q}}(\lambda)+\alpha_{\mathrm{u}}(t) F_{\mathrm{u}}(\lambda) \\
& +\alpha_{\mathrm{p}}(t) F_{\mathrm{p}}(\lambda)+\left[\alpha_{\mathrm{f}}(t)+\alpha_{\mathrm{n}}(t)\right] F_{\mathrm{f}}(\lambda) .
\end{aligned}
$$

Integration over all wavelengths gives the total solar irradiance.

\subsection{Parameters and optimization}

Our model, outlined in Sects. 2.1 and 2.2, contains a number of parameters, which are constrained by independent observations to lie within a given range rather than to a unique value. The main parameters suffering from such an uncertainty are $c_{x}$ and the amplitude factor, $X$, which relate the length and the amplitude of the ER cycles, respectively, to the corresponding values for the AR cycles. To a lesser extent the transfer time from AR to the open flux, $\tau_{\text {ta }}$, and the decay time of the open flux, $\tau_{\text {open }}$, which mainly influence the evolution of the open flux, are also affected. In some cases, as described in the next section, we also vary the saturation magnetic flux, $\phi_{\text {sat }}$, in order to test the sensitivity and the limits of our model. Table 1 provides an overview of all parameters, their values for the 4 models discussed below 

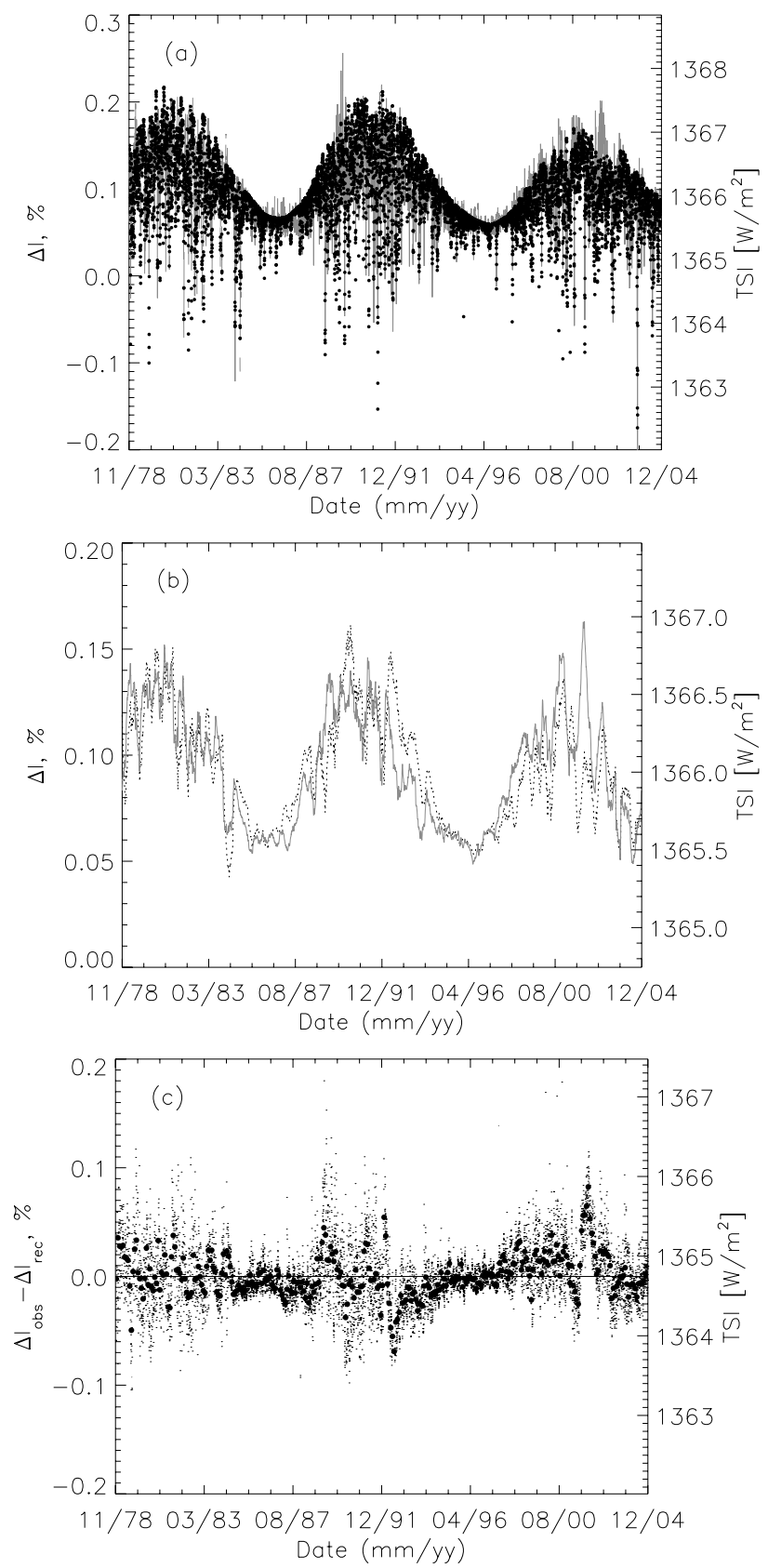

Fig. 4. Modelled (standard model; dots) and observed (PMOD composite, grey solid line) variations of the total solar irradiance in cycles 21-23. a) Daily values; b) 3-month runnning means; c) the difference between the model and the data (each dot represents a daily value, the bigger filled circles 30 day averages).

in more detail and the ranges within which the parameters have been varied.

We believe that it is important to constrain the output of the model using as many data sets as possible. Our model is tested against 3 independent data sets. Firstly, the reconstructed total irradiance should reproduce direct space based measurements which are available since 1978 . For a comparison with the model, we use the composite from the Davos Observatory (PMOD composite, Fröhlich 2006). Secondly, almost daily observations of the solar global photospheric field have been carried out at the Mt. Wilson Solar Observatory (MWO), KP NSO and Wilcox Solar Observatory (WSO) over cycles 21-23. Arge et al. (2002) present a compilation of these data, whereby they
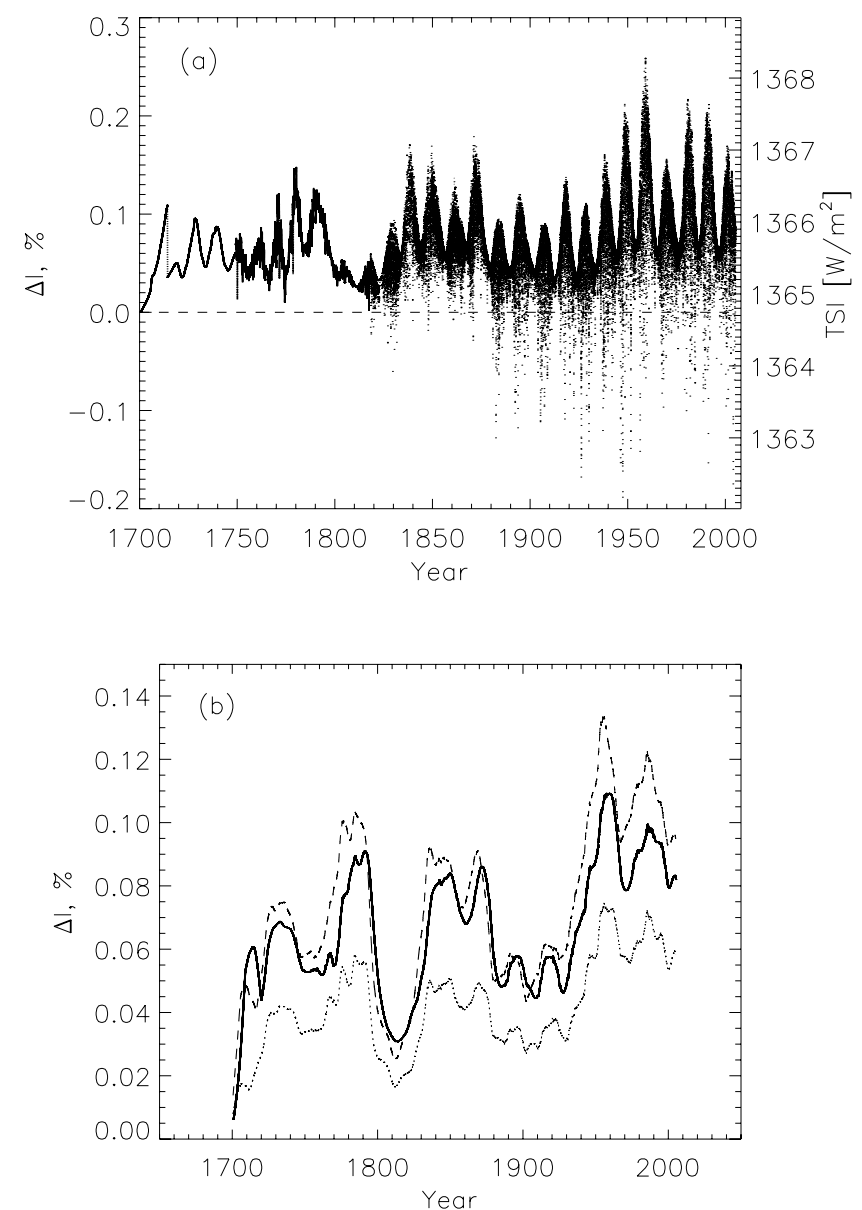

Fig. 5. a) Reconstructed TSI since 1700 for the standard model. b) $11-y r$ running mean of the reconstructed TSI for the standard (solid line), "antiphase (2)" (dashed) and "constant $\phi_{\mathrm{eph}}(2)$ " (dotted) models.

have introduced corrections to the older NSO KP data, which suffered from instrumental problems. This reduces the magnetic flux in cycle 21 to roughly the level in cycle 22 (cf. Wenzler et al. 2006). The total magnetic flux derived from these measurements can thus be compared to the modelled one. When doing so, we take into account the finding by Krivova \& Solanki (2004) that more than half of the photospheric flux from ER may go uncounted in the employed synoptic charts due to their relatively low spatial resolution. Therefore, the measured flux is compared to the value $\phi_{\text {act }}+0.4 \phi_{\text {eph }}+\phi_{\text {open }}$. Finally, the heliospheric magnetic flux has been reconstructed by Lockwood et al. (1999) based on the geomagnetic $a a$-index, and we compare the calculated open magnetic flux to their empirical reconstruction.

In order to find a solution which provides as close fits to all 3 sets of data as possible, we take advantage of the PIKAIA software (Charbonneau 1995) available from the ftp archive of the High Altitude Observatory: http://www.hao.ucar.edu. PIKAIA is a general purpose function optimization routine based on a genetic algorithm, which is particularly efficient in finding absolute exrema of multimodal optimization problems. We employ PIKAIA to find a set (or sets) of parameters minimizing the mean of $\chi^{2}$ values between the modelled and measured TSI, $\chi_{\mathrm{TSI}}^{2}$, total photospheric magnetic flux, $\chi_{\mathrm{TMF}}^{2}$, and solar open magnetic flux, $\chi_{\text {open }}^{2}$, all normalised by the number of points, $N$, 
in the corresponding data set. In other words, we search for the minimum of the mean variance:

$\sigma^{2}=\frac{1}{3}\left(\sigma_{\mathrm{TSI}}^{2}+\sigma_{\mathrm{TMF}}^{2}+\sigma_{\mathrm{open}}^{2}\right)$

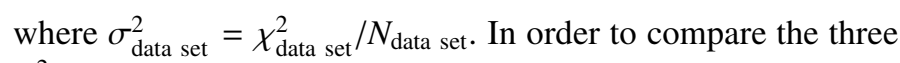
$\sigma^{2}$ values directly, we scale the total and open magnetic fluxes to the absolute level of the TSI before computing the appropriate $\sigma^{2}$.

\section{Results}

\subsection{Standard model}

We first ran PIKAIA by allowing the 4 main free parameters listed in Table 1 to vary within the ranges suggested by different observational data (see Sect. 2.1). The results for the model providing the smallest $\sigma^{2}$ are presented in Figs. 2-4: Fig. 2a compares the calculated total photospheric magnetic flux with the measurements since 1974, Fig. 3 the modelled open flux with the a $a$-based reconstruction of Lockwood et al. (1999) and Fig. 4 the reconstructed TSI with the PMOD composite. The estimated values of the free parameters as well as the $\sigma^{2}$ values for the TSI, $\sigma_{\text {TSI }}^{2}$, total magnetic flux, $\sigma_{\text {TMF }}^{2}$, and the open flux, $\sigma_{\text {open, }}^{2}$, are summarised in Table 1. Also given is the correlation coefficient, $R_{\mathrm{c}, \mathrm{TSI}}$, between the modelled and observed variations of the TSI.

Figure 2a shows the total photospheric magnetic flux for each Carrington rotation between 1974 and 2002 measured using the WSO (circles), NSO KP (triangles) and MWO (squares) synoptic charts and calculated with our model (solid curve). Figures $2 \mathrm{~b}$ and $2 \mathrm{c}$ illustrate the evolution of the magnetic flux of each photospheric component (AR, ER and the open flux) as well as their sum (total magnetic flux) since 1975 and 1700, respectively. Note that the effect of the spatial resolution on the visibility of the small ER is taken into account in Fig. 2a by considering the quantity $\phi_{\text {act }}+0.4 \phi_{\text {eph }}+\phi_{\text {open }}$ rather than just $\phi_{\text {act }}+\phi_{\text {eph }}+\phi_{\text {open }}$. The calculated curve lies in Fig. 2a well within the scatter of the data and reproduces both the amplitude of the variations and the cycle length quite well. In particular, the amplitudes of cycles 21 and 22 are comparable to each other whereas cycle 23 is somewhat weaker. Note that a similar comparison by Solanki et al. (2002) showed a strong disparity between the measurements (raw NSO KP data, without the corrections proposed by Arge et al. 2002) and the model in cycle 21 . As Fig. 2a shows, this disparity has now disappeared. It turns out that it was due to problems with the data rather than to a shortcoming of the model.

Since the open flux controlled in the model by the transfer time from $\mathrm{AR}, \tau_{\text {ta }}$, and the decay time, $\tau_{\text {open }}$, contributes rather little to the total flux $(\approx 10-15 \%$, see Figs. $2 \mathrm{~b}$ and $2 \mathrm{c})$, the main two parameters determining the evolution of the total photospheric magnetic flux are $c_{x}$ and $X$, relating the length and the amplitude of the ER cycles, respectively, to the corresponding values for the AR cycles. Encouragingly, values of $c_{x}=7.6 \mathrm{yr}$ and $X=117$ found by the optimization procedure lie within the respective limits suggested by observations.

Since the ER cycles partly overlap, the ER flux varies much less over the cycle than the AR flux, only by approximately a factor of 2 (see Figs. $2 b$ and 2c). Thus, whereas the flux in ER is only slightly lower than that in AR at activity maxima (cf. Krivova \& Solanki 2004), it clearly dominates at minima. For recent cycles (Fig. 2b), the minimum flux in ER is reached slightly $(\approx 1-2$ years) before sunspot minimum (note that, in contrast to the model described in Sect. 3.2, no shift is imposed here, see Eq. (6)). All this is in good agreement with the conclusions of Harvey $(1993,1994 b)$.

The decay time of the open flux, $\tau_{\text {open }}$, only enters Eq. (3) and the transfer time from AR to the open flux, $\tau_{\text {ta }}$, is significantly longer ( $\gtrsim 10 \mathrm{yr})$ than the decay times of AR and ER ( $\sim 3$ months and $14 \mathrm{~h}$, respectively). Thus, as mentioned above, these two parameters mainly affect the evolution of the open flux component (see Fig. 3). Solanki et al. (2000) modelled the evolution of the open flux considering only AR and obtained values of $\tau_{\text {ta }}=12 \mathrm{yr}$ and $\tau_{\text {open }}=4 \mathrm{yr}$ from a comparison with the reconstruction by Lockwood et al. (1999). Inclusion of ER led to a slightly lower value of $\tau_{\text {open }}=3 \mathrm{yr}$ (Solanki et al. 2002), although it should be noted that the contribution of ER to the open flux is rather uncertain. The values we obtain with the standard model, $\tau_{\mathrm{ta}}=13.5 \mathrm{yr}$ and $\tau_{\text {open }}=3.5 \mathrm{yr}$ are close to the previously obtained values (cf. Lockwood 2003).

The modelled (dots) and measured (grey solid line; PMOD composite by Fröhlich 2006) variations of the total solar irradiance are compared in Fig. 4. Figure 4a shows daily values, Fig. 4b the 3-month running means and Fig. 4c the difference between the model and the data (dots mark daily values and the bigger filled circles the 30-day averages). Similarly to the total magnetic flux, the modelled TSI is mainly driven by the 2 parameters, $c_{x}$ and $X$ describing the ER cycle (the length and the amplitude) via characteristics of the corresponding AR cycle (known from the historical sunspot record). Obviously, the coarse model used to calculate the evolution of the magnetic flux on the solar surface is not designed to match the observed shorter-term variations down to the last detail. In particular, the sine approximation of the shape of the ER cycle is relatively rough, which is clearly seen during the ascending and descending phases of cycle $22^{1}$. Nevertheless, both the amplitude and the phase of the variations are adequately reconstructed and the agreement between the model and the observations is fairly good with a correlation coefficient of $R_{\mathrm{c}}=0.78$. Both the short-term (rotation time scale) and longer-term (solar cycle) variations are equally well reproduced.

We now use the same model to calculate the variations of the TSI since the end of the Maunder minimum. The reconstructed irradiance since 1700 is presented in Fig. 5 (based on the Zurich sunspot number). The quality of the reconstruction improves with time: only yearly values of the sunspot number (and thus of the resulting TSI) are available before 1749, monthly averages can be calculated for the period between 1749 and 1818 , whereas daily sampling is possible after 1818 (see panel a of Fig. 5). The longer-term trend is better evident in panel $b$ of this figure, where an 11-year running mean of the reconstructed TSI is shown (solid line). Thus if we require that the model agrees as well as possible with all available observational data, it predics an increase in the cycle averaged TSI of about $0.095 \%$ or $1.3 \mathrm{Wm}^{-2}$ between the end of the Maunder minimum and the present (where present-day level is taken as an average over last 30 years). Note that this estimate of the secular change is fairly stable in the sense that if we vary the free parameters within the reasonable ranges, we typically arrive at values close to those listed above (within about $\pm 0.2-0.3 \mathrm{Wm}^{-2}$ ). Below we discuss two extreme cases in more detail. It is also worth noting that none of the reasonable combinations of the parameters led to a

\footnotetext{
1 We also tried using the shape of the (correspondingly stretched) sunspot cycle for the ER cycle. This did not improve the correspondence of the modelled to the observed irradiance.
} 

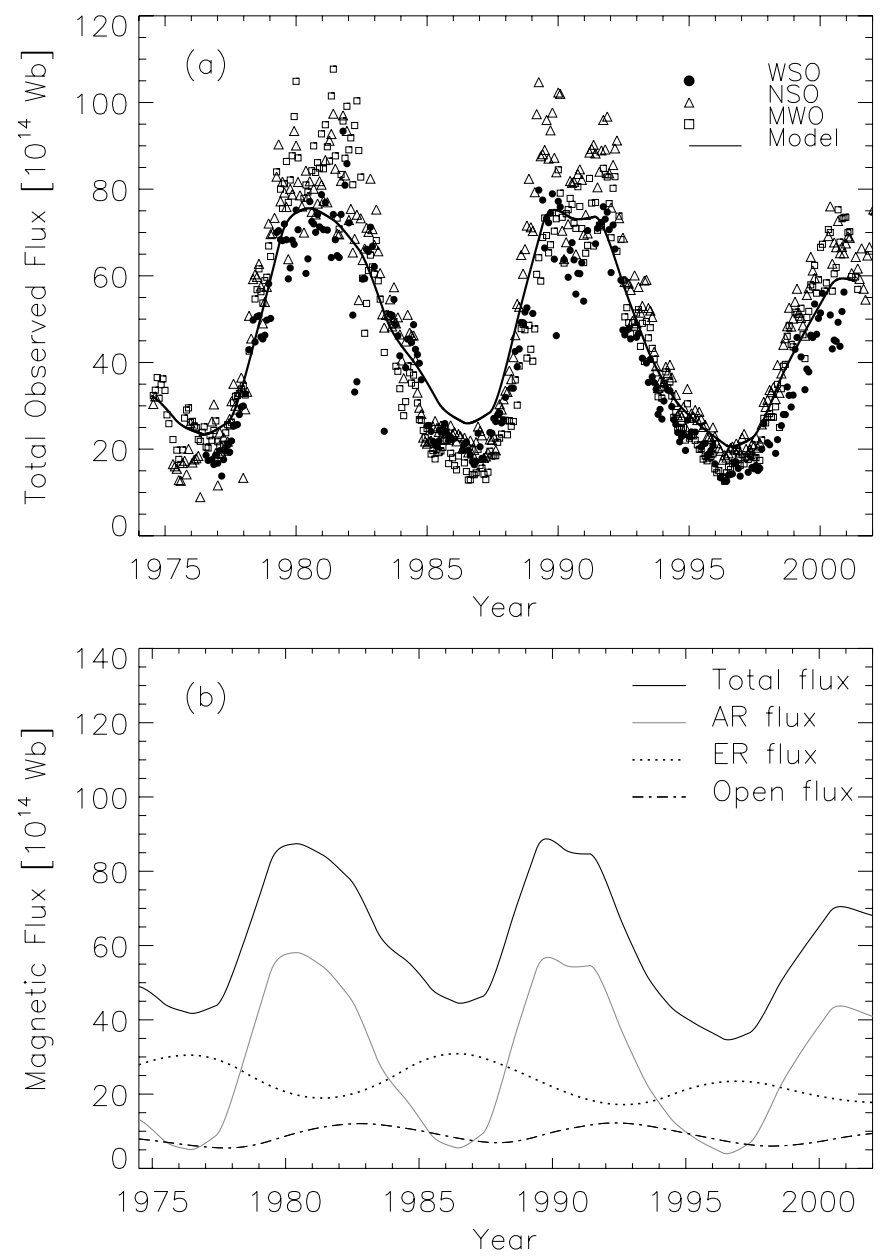

Fig. 6. Same as Figs. $2 \mathrm{a}$ and $\mathrm{b}$ but for the model with ER running 5 years ahead of AR (model "antiphase (2)" in Table 1).

higher level of the irradiance at the minimum of cycle 23 than at the minimum of cycle 22 as proposed by Willson (1997).

\subsection{A possible shift between the ER and $A R$ evolution}

The main uncertainity in our model is introduced by the unknown temporal evolution of the emergence rate of the ER. Although it is evidently related to the emergence rate of AR, the exact form of this relationship is not well established. Based on the observational evidence outlined in Sect. 2.1, we assume that this relationship is described by Eqs. (5)-(8), which implies that although ER of a new cycle appear earlier on the solar surface than the corresponding AR cycle starts, the two components still evolve nearly in phase. Hagenaar et al. (2003) found, however, that the number density of emerging ER decreased by about a factor of 1.5 from 1997 (i.e. right after the sunspot minimum in 1996) to a minimum in 2000 (when the sunspot number reached its maximum). They concluded thus that the two components may progress in antiphase, or at least with a significant shift. Therefore we now investigate this possibility.

We intoduced a shift between the emergence rates of AR and ER, with ER always preceding the AR. This shift was allowed to be between 2 and 8 years and was thus an additional parameter. A point that should be mentioned is that no good solution matching reasonably all 3 data sets (with TSI being the most critical) was initially found (see Table 1, model "antiphase(1)"). The contribution of ER to the total magnetic flux turned to be too
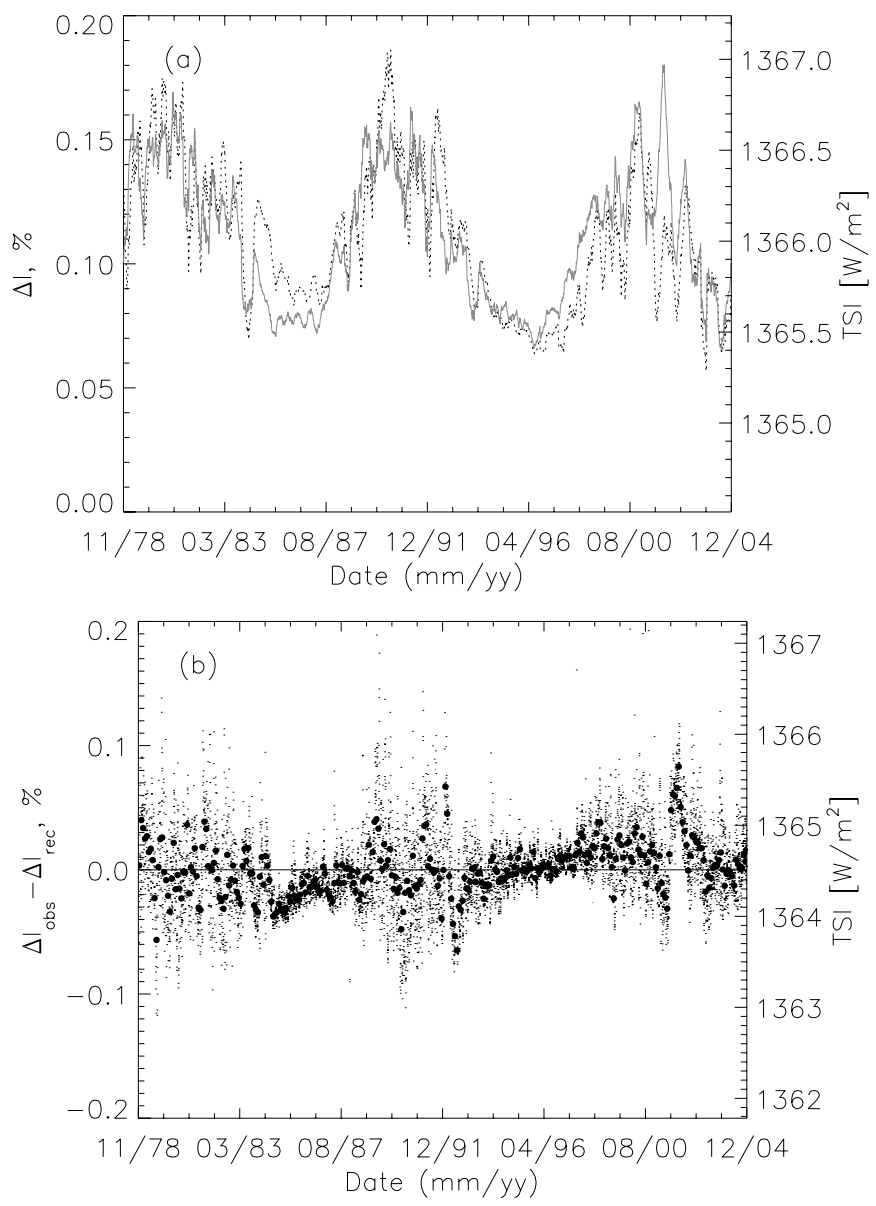

Fig. 7. Same as Figs. $4 \mathrm{~b}$ and $4 \mathrm{c}$ but for the model with ER running 5 years ahead of AR (model "antiphase (2)" of Table 1).

strong, in particular during activity minima, and led to a heavily deformed shape of the irradiance cycles.

Only after introducing an additional parameter, i.e. after allowing one previously fixed parameter to vary, could reasonable fits be obtained. This new parameter is the saturation flux

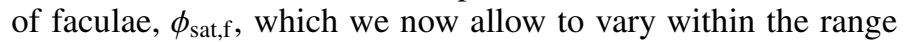
150-400 G (see Table 1). Indeed, the value of $300 \mathrm{G}$ that we use for faculae was adopted from our reconstructions based on MDI and KP NSO magnetograms with no disctinction between faculae and the network. Since the value for the network saturation flux is somewhat higher than this (see Sect. 2.2), the value of $\phi_{\text {sat, f }}$ can, in principle, be somewhat (although hardly significanly) lower.

The best fit was obtained with $\phi_{\text {sat, } \mathrm{f}}=168 \mathrm{G}$ (see Table 1 , model "antiphase (2)"). This low value indicates that the facular contribution to the cyclic variation needs to be strongly amplified in this model, in order to compensate for the contribution of ER evolving in antiphase with the solar cycle (see Fig. 6b). Also, the ER amplitude parameter, $X$, dropped down to 80 , which is the lowest possible value within the allowed range. This means that better fits are obtained when the antiphase variation of ER is dampened down. A shift of 5 years was found to be in best agreement with the observed records, which implies that ER and AR evolve nearly in antiphase. Note, however, that if a shift of 0 is also allowed, then the standard model is selected as the one providing the best possible fits to the data (with the smallest number of free parameters). In order to match the reconstruction of the heliospheric magnetic flux by Lockwood et al. (1999), we also had to increase the transfer time from AR to the open 

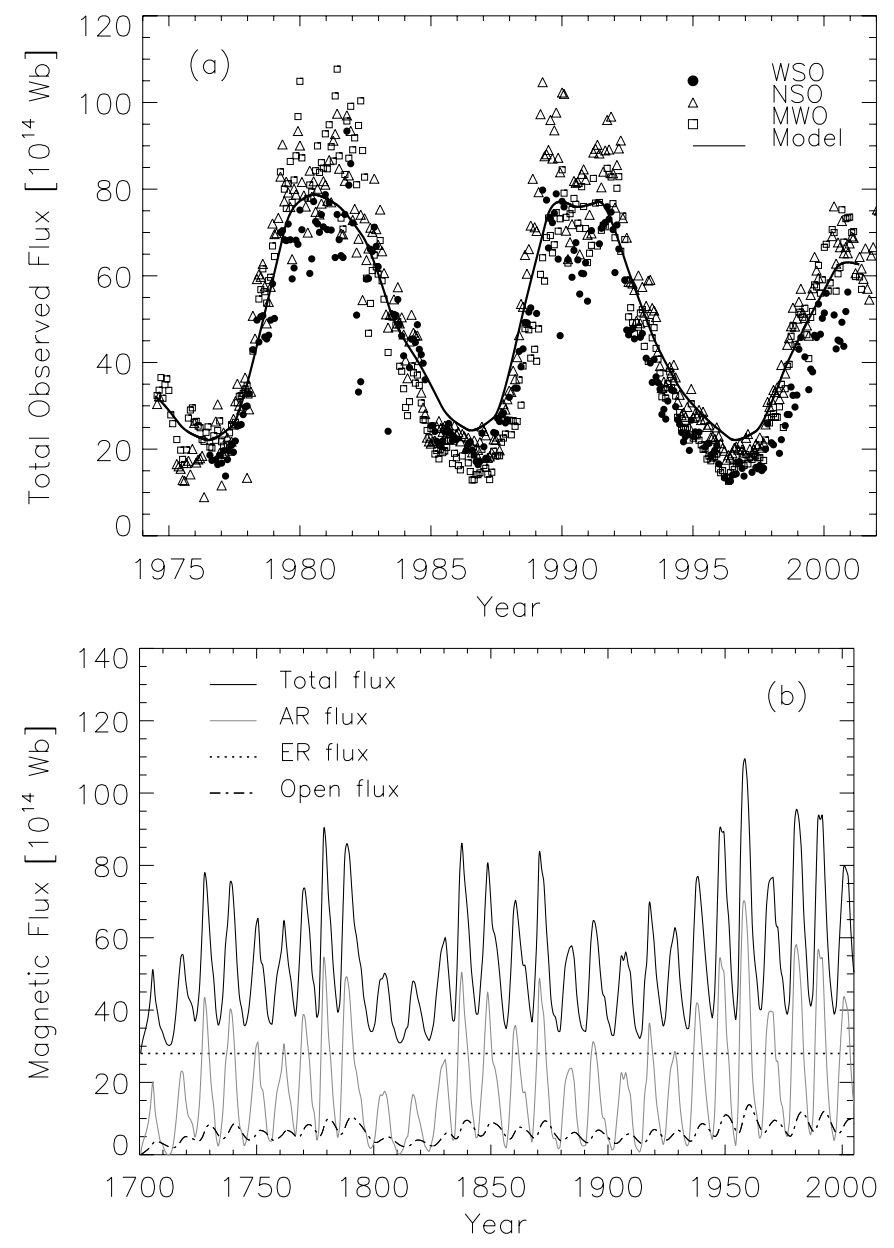

Fig. 8. Same as Figs. 2a and 2c but for the model with the constant ER magnetic flux, $\phi_{\mathrm{eph}}=2.8 \times 10^{15} \mathrm{~Wb}$ ("const. $\phi_{\mathrm{eph}}(2)$ " in Table 1).

flux, $\tau_{\mathrm{ta}}=13.9$ years, and the decay time of the open flux, $\tau_{\text {open }}=3.9$ years.

The model with these parameters reproduces the total photospheric magnetic flux measured in cycles 21-23 less well than the standard model (see Fig. 6a and Table 1). Particularly, the flux at the minima preceding cycles 22 and 23 appears too high compared to the observations due to the ER contribution as demonstrated by Fig. 6 b.

The agreement of the reconstructed irradiance with the measured is also worse than in the standard model (Fig. 7 and Table 1). Specifically, the solar cycle trend in the model systematically lags behind that in the data by about a year. This concerns only the overall irradiance level, while the shorter-term (rotational time scales) changes due to AR occur simultaneously in the model and the data. The shift is probably due to the phase shift in the ER emergence rate, while the good short-term agreement is due to the active regions, in particular the sunspots. For the open flux, the results are rather similar in both cases, with $\sigma_{\text {open }}^{2}$ values being even somewhat smaller in the "antiphase (2)" model (Table 1). Note, however, that the antiphase model has 2 additional free parameters and the value of $\phi_{\mathrm{sat}, \mathrm{f}}=168 \mathrm{G}$ required to reproduce the observed irradiance variations seems to be unreasonably low.

Although our preference is given to the standard model for the reasons outlined above, we cannot rule the antiphase model out and, in order to evaluate the uncertainty in the estimated secular change in the irradiance, we have reconstructed the TSI
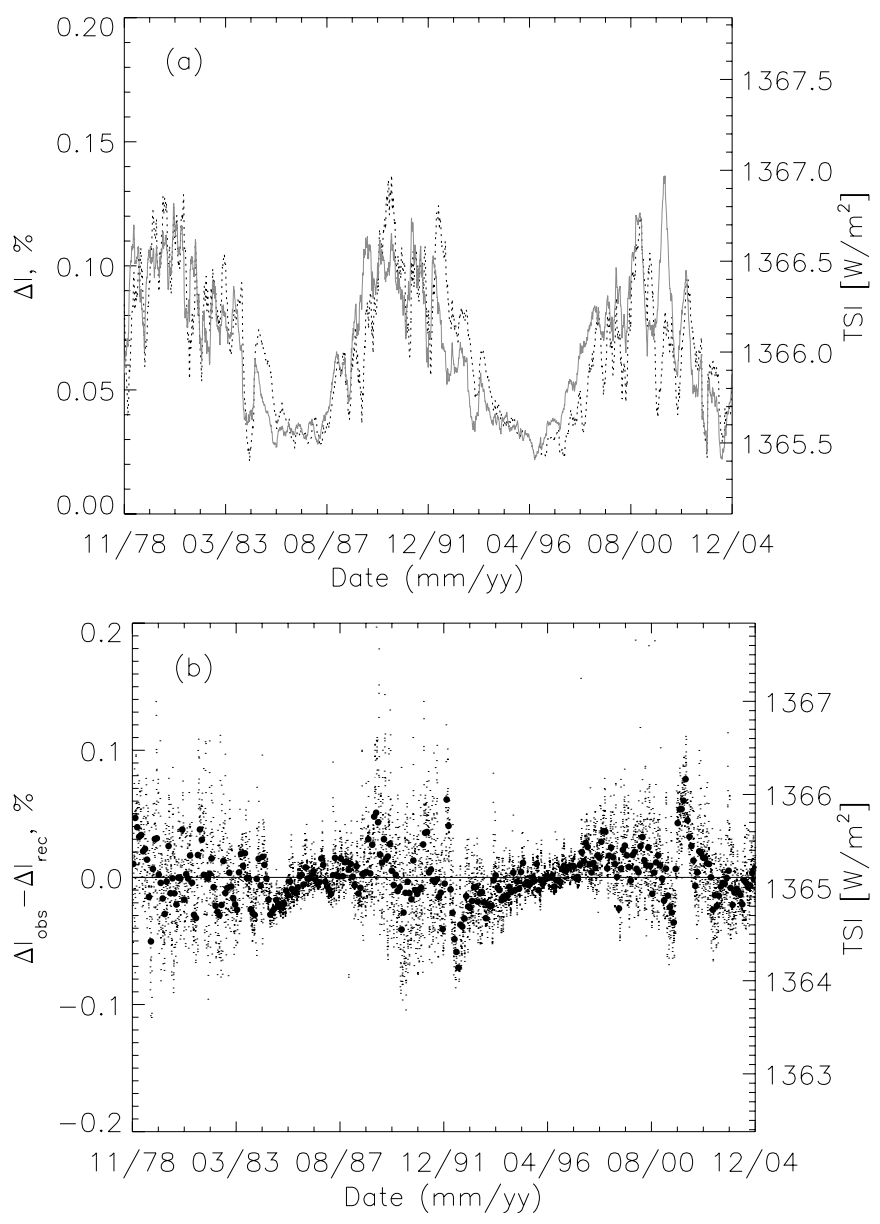

Fig. 9. Same as Figs. $4 \mathrm{~b}$ and $4 \mathrm{c}$ but for the model with the constant ER magnetic flux, $\phi_{\text {eph }}=2.8 \times 10^{15} \mathrm{~Wb}$ ("const. $\phi_{\text {eph }}(2)$ " in Table 1).

back to 1700 using this model as well. It is shown in Fig. 5b by the dashed line. The predicted increase in the cycle-averaged TSI since that time is about $0.11 \%$ or $1.5 \mathrm{Wm}^{-2}$, which is $15 \%$ higher then the value obtained with the standard model (see Table 1).

\subsection{Constant ER flux}

Observations suggest that during cycles 21-23 the emergence rate and the total magnetic flux of ER varied rather little, by no more than a factor of 2 and it might well be that this factor is very close to 1 (Harvey 1993, 1994b; Hagenaar et al. 2003). Therefore we now also consider the important special case with no variation in the ER flux at all. If the magnetic flux of the socalled quiet Sun (i.e. the ER flux) does not change, then the only source of a possible secular variation remains the open flux, beyond variations in amplitude from one cycle to the next. In this way we should obtain a lower limit to the increase of the TSI since the end of the Maunder minimum.

Since the quiet Sun magnetic flux is not known accurately, it is considered to be another free parameter. It replaces the two parameters, $c_{x}$ and $X$, used to describe the evolution of the ER flux with time. The facular saturation flux, $\phi_{\text {sat, }}$, also needs to be allowed to vary in order to obtain good fits (cf. models "const. $\phi_{\mathrm{eph}}(1)$ " and "const. $\phi_{\mathrm{eph}}(2)$ " in Table 1). Thus the number of free parameters in this model is the same as in the standard case.

The results for this model are shown in Figs. 8-9. The evolution of the total magnetic flux is reproduced less well by this model than by the standard model (cf. Table 1), and the flux is 

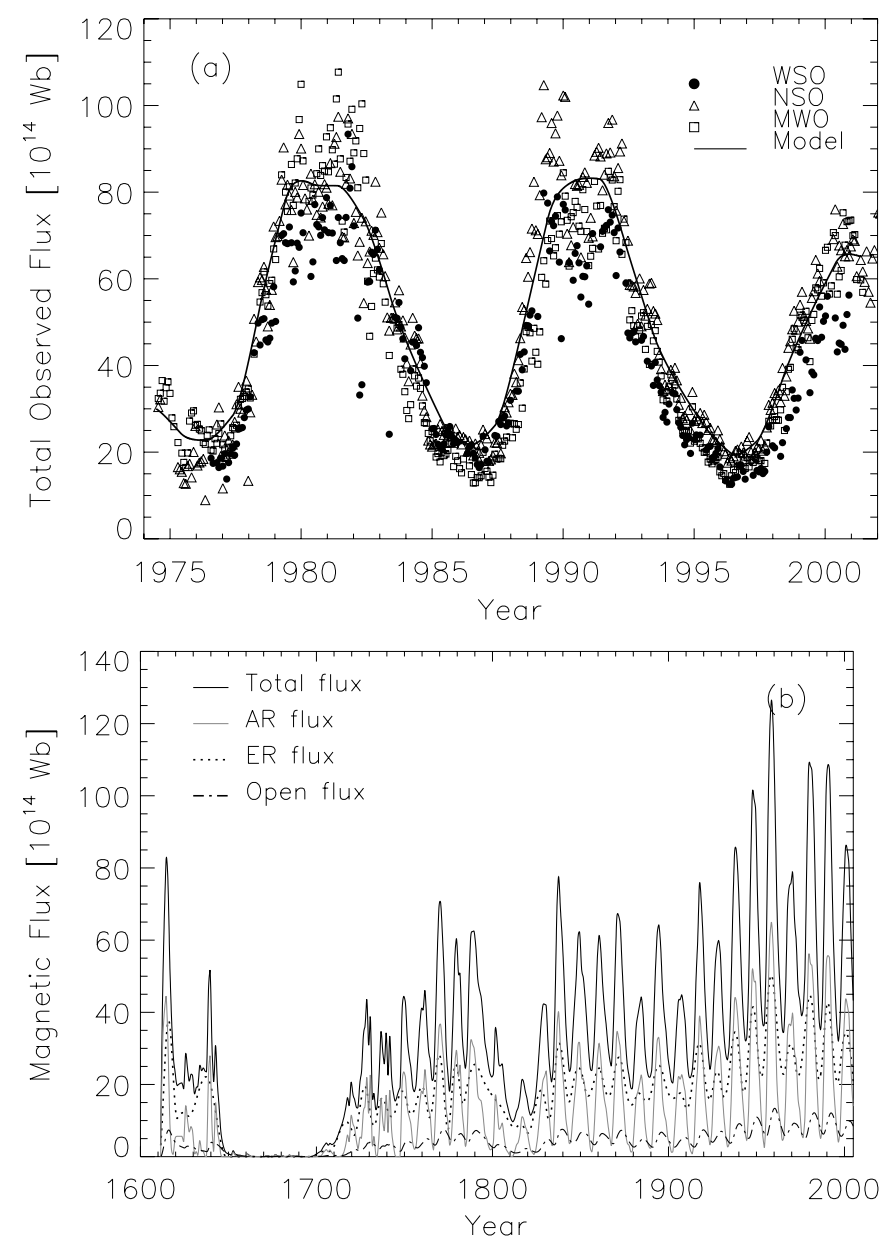

Fig. 10. Same as Figs. 2a and 2c but for the model based on $R_{\mathrm{G}}$.

rather high during activity minima (Fig. 8). The variations of the TSI are reproduced slightly (although not significantly) less well than by the standard model (Table 1 and Fig. 9).

In order to put a lower limit on the secular change in the TSI since 1700 , we utilise this model too to reconstruct irradiance variations back in time. The 11-year running mean of this reconstruction is shown by the dotted line in Fig. 5b. As expected, the resulting increase in the cycle averaged TSI is somewhat lower than in the other cases, $0.9 \mathrm{Wm}^{-2}$ or $\approx 0.065 \%$ (see also Table 1 ), but is still noticeable. As seen from Fig. 8b, this increase has 2 sources: the large amplitude of recent AR cycles and a secular rise in the open flux.

\subsection{Reconstruction based on the Group sunspot number}

The reconstructions carried out in previous sections were based on the Zurich sunspot number, $R_{\mathrm{Z}}$, available since $1700 . R_{\mathrm{Z}}$ is calculated using the number of both the individual sunspots and the sunspot groups present on the solar disc. In contrast, the Group sunspot number, $R_{\mathrm{G}}$ (Hoyt \& Schatten 1998) takes into account only the number of sunspot groups and goes back to 1610 , covering the whole Maunder minimum. A weighting factor was introduced by Hoyt \& Schatten (1998), in order to scale the Group sunspot number to the Zurich number for the period 1874-1976. Prior to 1874, however, the Zurich number is, on average, higher than the Group number.

Using the Group sunspot number it is possible to reconstruct solar magnetic flux and solar irradiance for a period of more

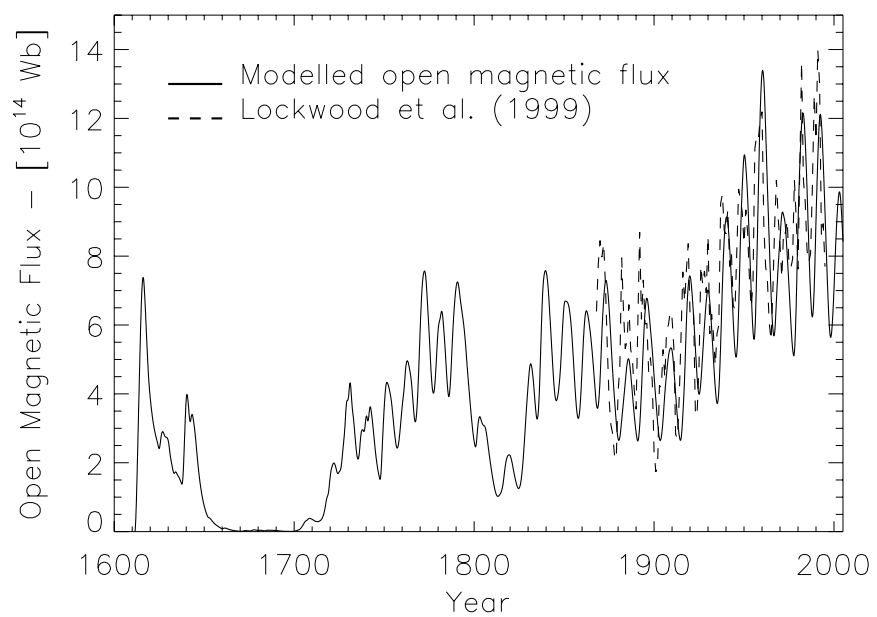

Fig. 11. Same as Fig. 3 but for the model based on $R_{\mathrm{G}}$.
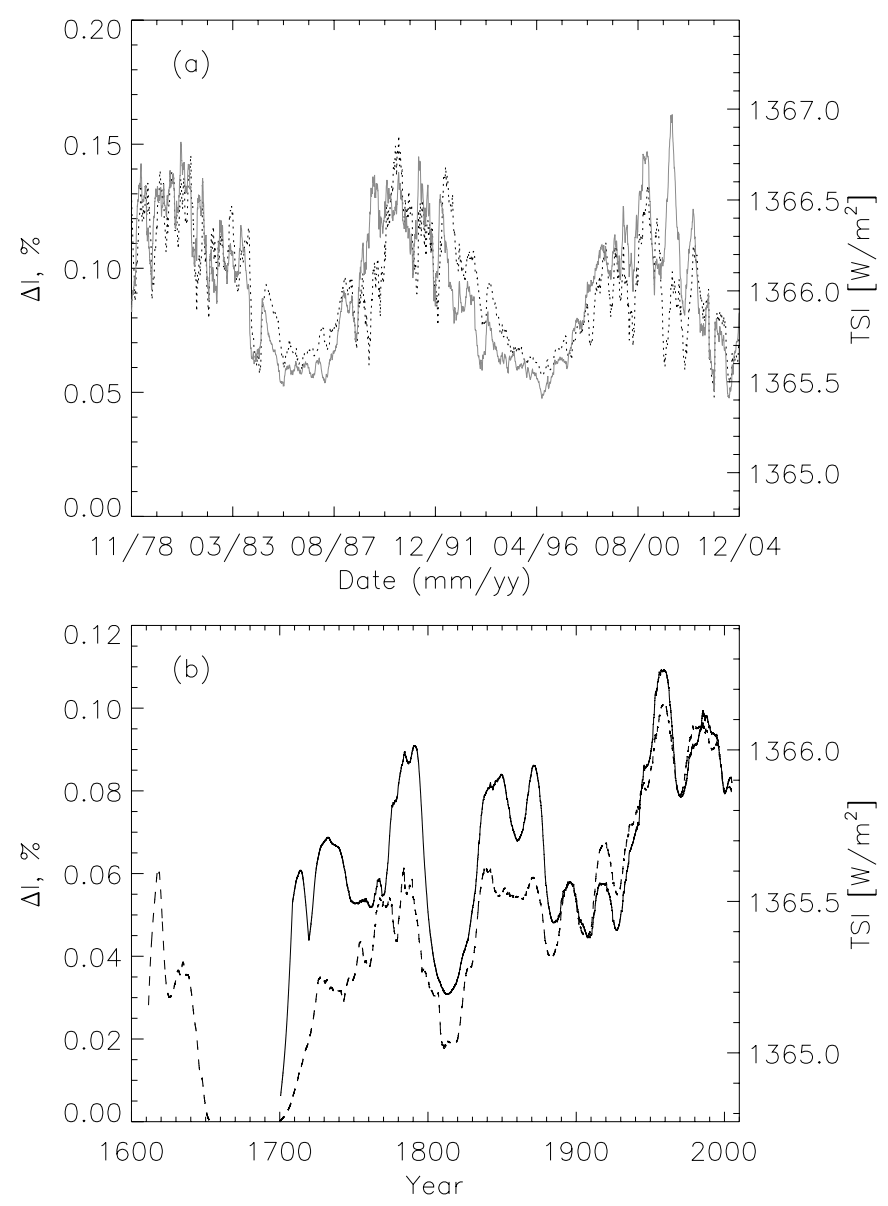

Fig. 12. Same as Figs. $4 \mathrm{~b}$ and $4 \mathrm{c}$ but for the model based on $R_{\mathrm{G}}$.

than 36 cycles, thus extending the model to the beginning of the Maunder minimum. We first ran PIKAIA using $R_{\mathrm{G}}$ instead of $R_{\mathrm{Z}}$, and the 4 main free parameters were allowed to vary within the same ranges as listed in Table 1. The obtained set of parameters turned to be very similar to the one obtained in Sect. 3.1 with the Zurich sunspot number, $R_{\mathrm{Z}}$, and the $\sigma^{2}$ values changed only imperceptably. This is due to the normalisation introduced by Hoyt \& Schatten (1998) for the period after 1874. Therefore, for the $R_{\mathrm{G}}$-based reconstruction we opt for the same set of values as in the standard $R_{\mathrm{Z}}$ model. 

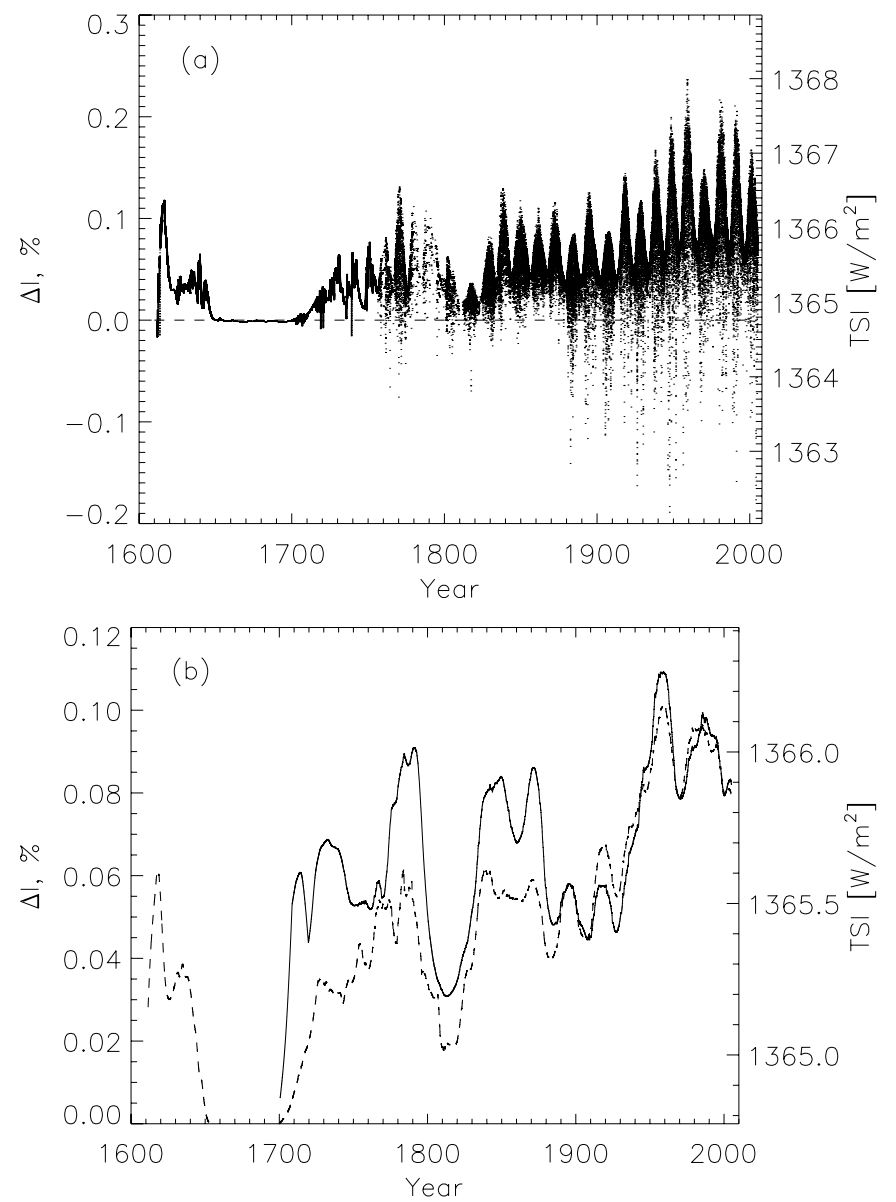

Fig. 13. a) Reconstructed irradiance variations since 1610 for the standard model based on the Group sunspot number, $R_{\mathrm{G}}$. b) 11-yr running mean of the reconstructed TSI for the standard models based on the Zurich (solid line) and Group (dashed) sunspot numbers.

The reconstructed total magnetic flux is compared to the data from different observatories in Fig. 10a. The model reproduces both the cycle amplitude and cycle length of the observed magnetic flux, and the fit is about as good as in the case of the standard $R_{\mathrm{Z}}$ model (cf. Fig. 2a and Table 1). The evolution of the magnetic flux in active regions, ephemeral regions as well as the open and total magnetic flux are shown in Fig. 10b.

During the Maunder minimum the sunspot number was extremely low, with extended periods without any sunspots at all. Therefore the magnetic flux in active regions dropped almost to zero. In our model, the evolution of the other two components of the magnetic flux (i.e. ER and open flux) is related to the evolution of the flux emerging in active regions. Thus flux in these components drops to zero as well. Of course, the lack of sunspots does not necessarily imply that also smaller bipolar regions stopped emerging at the solar surface. E.g., the ${ }^{10} \mathrm{Be}$ data suggest that the open flux showed periodic fluctuations during the Maunder minimum (Beer et al. 1998). Unfortunately, an estimate of the amount of flux emerging on the solar surface during this period is not straightforward and we assume for simplicity that all components of the magnetic flux drop to zero during the Maunder minimum.

In Fig. 11 the modelled open flux (solid line) is compared to that reconstructed by Lockwood et al. (1999) from the $a a$-index (dashed). Note that this component of the magnetic flux is better reproduced using the Group sunspot number (see Table 1).
In Fig. 12 the TSI reconstructed using $R_{\mathrm{G}}$ (dots) is compared to the PMOD composite of space measurements (solid line). Although the $\sigma_{\text {TSI }}^{2}$ values are very similar for the models based on $R_{\mathrm{Z}}$ and $R_{\mathrm{G}}$, the TSI reconstructed with $R_{\mathrm{G}}$ is somewhat higher than observed in the minima preceding cycles 22 and 23 , and the corresponding correlation coefficient, $R_{\mathrm{c}}$, is lower ( 0.75 vs. 0.78 for the standard $R_{\mathrm{Z}}$ model). This may be due to the fact, that by ignoring individual spots, $R_{\mathrm{G}}$ contains less information about the evolution of the weaker fields than $R_{\mathrm{Z}}$, and is thus a worse proxy of the ER evolution. At the same time, the open flux is well described by the large-scale magnetic field and is well represented by $R_{\mathrm{G}}$.

The long-term evolution of the solar irradiance since 1610 is shown in Fig. 13a. Note that it is almost identical with the reconstruction using the values of the free parameters obtained by running PIKAIA. In Fig. 13b, the 11-yr running mean of this reconstruction (dashed line) is plotted together with that obtained with the $R_{\mathrm{Z}}$ standard model (solid). This figure shows that during the period 1700-1874 the irradiance reconstructed from the Zurich sunspot number is higher than the one obtained from the Group number. This is because $R_{\mathrm{Z}}$ is higher than $R_{\mathrm{G}}$ at that time. The change in the TSI since the Maunder minimum is, however, almost the same in both cases, $1.3 \mathrm{Wm}^{-2}$ (the difference between the 2 values is about $1.5 \%$ and is statistically insignificant).

\section{Conclusions}

Using the physical model of Solanki et al. (2002) to calculate the evolution of the solar photospheric magnetic flux we have reconstructed solar total irradiance back to 1700 (or even 1610 if the Group sunspot number is employed). In this model, the cyclic variation of ephemeral regions (ER) is related to the properties of the active region (AR) cycles, which are represented by the historical records of the sunspot number and sunpot areas. The change in the level of the quiet Sun magnetic flux caused by the partial overlap of the ER cycles leads to a secular variation in the solar total magnetic flux and irradiance.

Our basic model has 4 free parameters. They are estimated by fitting simultaneously 3 independent time series which are the total photospheric magnetic flux in cycles 21-23, the heliospheric magnetic flux since 1868 empirically reconstructed by Lockwood et al. (1999) and the space-borne measurements of the TSI since 1978. Two of the free parameters, the transfer time from AR to the open flux, $\tau_{\text {ta }}$, and the decay time of the open flux, $\tau_{\text {open }}$, mainly influence the evolution of the open flux and do not affect the total magnetic flux and irradiance. The 2 others, $c_{x}$ and $X$, link the properties of the ER cycles to those of the AR cycles and control the evolution of the ER magnetic flux (which is a significant part of the total photospheric flux) and the irradiance variations. The obtained values of these parameters are in good agreement with independent observational estimates.

Both historical sunspot records, the Zurich and Group sunspot numbers, have being employed. The irradiance reconstructed from the Group sunspot number is on average somewhat lower during 1700-1874. However, the predicted secular increase in the cycle averaged TSI since 1700 is almost the same in both models and is about $1.3 \mathrm{Wm}^{-2}$. In order to understand the reliability and uncertainty of this estimate we also consider two extreme cases, one assuming that ER progress in antiphase with $\mathrm{AR}$, and one assuming that the ER magnetic flux does not change in time.

Since both of these models initially produced noticeably worse fits to, at least, one of the considered independent time 
series, we extended the set of free parameters. Then, fairly good fits were obtained for these alternative models. The reconstructed TSI since 1700 looks quite similarly for all the cases (see Fig. 5b). The model with the ER cycles being in antiphase with those of the AR provides the highest rise in the cycle averaged TSI of about $1.5 \mathrm{Wm}^{-2}$, whereas the model with a constant ER flux puts a lower limit of about $0.9 \mathrm{Wm}^{-2}$ to this quantity.

This low value is in agreement with findings of Foster (2004); Lockwood (2005) and Wang et al. (2005). This is not surprising. Wang et al. (2005) neglected the extended length of the ephemeral region cycle. Therefore, the increase in TSI since the Maunder minimum they find $\left(1 \mathrm{Wm}^{-2}\right)$ is expected to be similar to that which we obtain when we do not allow the ephemeral region flux to change with time. Foster (2004), cf. Lockwood (2005), did take the network into account and obtained an upper limit of $1.7 \mathrm{Wm}^{-2}$ on the TSI change by assuming that the network disappeared during the Maunder minimum. This is close to the value of $1.5 \mathrm{Wm}^{-2}$ we obtain for our extreme model showing the largest $\triangle$ TSI. They then assume that half of the network was still present in the Maunder minimum, which gives a $\Delta \mathrm{TSI} \approx 1 \mathrm{Wm}^{-2}$. This lies between the values of $\approx 0.9 \mathrm{Wm}^{-2}$ and $\approx 1.3 \mathrm{Wm}^{-2}$ obtained for our constant ER flux and standard models. The $\triangle$ TSI obtained in this paper thus cover the range found from independent recent reconstructions. However, all the values we obtain are significantly below the $\Delta$ TSI values deduced from stellar data and used in older TSI reconstructions.

Acknowledgements. We are grateful to M. Schüssler for providing the code for simulations of the magnetic flux evolution and helpful discussions. We thank C. N. Arge, J. W. Harvey and H. P. Jones for providing the photospheric magnetic flux data. The PIKAIA optimization subroutine used in this work is available from http://download.hao.ucar.edu/archive/pikaia/. Historical sunspot data were obtained from the National Geophysical Data Center (NGDC, http://www.ngdc.noaa.gov/stp/SOLAR/solar.html). This work was supported by the Deutsche Forschungsgemeinschaft, DFG project number SO 711/1-1.

\section{References}

Arge, C. N., Hildner, E., Pizzo, V. J., \& Harvey, J. W. 2002, J. Geophys. Res., 107 (A10), 16

Arnold, N. 2005, Solar variability, coupling between atmospheric layers and climate change, ed. J. M. T. Thompson (London: Imperial College Press), 391

Baliunas, S., \& Jastrow, R. 1990, Nature, 348, 520

Balmaceda, L., Solanki, S. K., \& Krivova, N. 2005, Mem. Soc. Astron. It., 76, 929

Beer, J., Tobias, S., \& Weiss, N. 1998, Sol. Phys., 181, 237

Bond, G., Kromer, B., Beer, J., et al. 2001, Science, 294, 2130

Brueckner, G. E., Edlow, K. L., Floyd, L. E., Lean, J. L., \& Vanhoosier, M. E. 1993, J. Geophys. Res., 98, 10695

Charbonneau, P. 1995, ApJS, 101, 309

Coughlin, K., \& Tung, K. K. 2004, J. Geophys. Res. (Atmospheres), 109, 21105

Dameris, M., Matthes, S., Deckert, R., Grewe, V., \& Ponater, M. 2006, Geophys. Res. Lett., 33, 3806

Eddy, J. A. 1976, Science, 192, 1189

Egorova, T., Rozanov, E., Zubov, V., Schmutz, W., \& Peter, T. 2005, Adv. Sp. Res., 35, 451

Fligge, M., \& Solanki, S. K. 2000, Geophys. Res. Lett., 27, 2157

Floyd, L., Rottman, G., DeLand, M., \& Pap, J. 2003, ESA SP-535, 195

Foster, S. 2004, Ph.D. Thesis, University of Southhampton, School of Physics and Astronomy

Foukal, P., \& Lean, J. 1990, Science, 247, 556

Fröhlich, C. 2003, ESA SP-535, 183

Fröhlich, C. 2006, Space Sci. Rev., 125, 53

Fröhlich, C., \& Finsterle, W. 2001, in Recent Insights Into the Physics of the Sun and Heliosphere - Highlights from SOHO and Other Space Missions, ed. P. Brekke, B. Fleck, \& J. B. Gurman, ASP Conf. Ser., 203, 105
Hagenaar, H. J. 2001, ApJ, 555, 448

Hagenaar, H. J., Schrijver, C. J., \& Title, A. M. 2003, ApJ, 584, 1107

Haigh, J. D. 1996, Science, 272, 981

Haigh, J. D. 1999, J. Atm. Terr. Phys., 61, 63

Haigh, J. D. 2001, Science, 294, 2109

Hall, J. C., \& Lockwood, G. W. 2004, ApJ, 614, 942

Harvey, K. L. 1992, in The Solar Cycle, ASP Conf. Ser., 27, 335

Harvey, K. L. 1993, Ph.D. Thesis, Univ. Utrecht

Harvey, K. L. 1994a, in Solar Surface Magnetism, ed. R. J. Rutten \& C. J. Schrijver (Dordrecht: Kluwer), 347

Harvey, K. L. 1994b, in The Sun as a Variable Star: Solar and Stellar Irradiance Variations, ed. J. M. Pap, C. Fröhlich, H. S. Hudson, \& S. K. Solanki (Cambridge: Cambridge Univ. Press), IAU Coll., 143, 217

Hoyt, D. V., \& Schatten, K. H. 1993, J. Geophys. Res., 98, 18895

Hoyt, D. V., \& Schatten, K. H. 1998, Sol. Phys., 179, 189

Keppens, R., \& Martínez Pillet, V. 1996, A\&A, 316, 229

Kodera, K., \& Kuroda, Y. 2002, J. Geophys. Res. (Atmospheres), 107 (D24), DOI 10.1029/2002JD002224

Krivova, N. A., \& Solanki, S. K. 2004, A\&A, 417, 1125

Krivova, N. A., \& Solanki, S. K. 2005, Mem. Soc. Astron. It., 76, 834

Krivova, N. A., Solanki, S. K., Fligge, M., \& Unruh, Y. C. 2003, A\&A, 399, L1

Kuroda, Y., \& Kodera, K. 2005, Geophys. Res. Lett., 32, 13802

Langematz, U., Matthes, K., \& Grenfell, J. L. 2005, Mem. Soc. Astron. It., 76, 868

Lean, J., Skumanich, A., \& White, O. 1992, Geophys. Res. Lett., 19, 1595

Lean, J., Beer, J., \& Bradley, R. 1995, Geophys. Res. Lett., 22, 3195

Lean, J. L., White, O. R., Livingston, W. C., \& Picone, J. M. 2001, J. Geophys. Res., 106, 10645

Lockwood, M. 2003, J. Geophys. Res., 108 (A3), 1128

Lockwood, M. 2005, in The Sun, Solar Analogs and the Climate, 34th Saas Fee Advanced Course, ed. I. Rüedi, M. Güdel, \& W. Schmutz (Berlin: Springer), 109

Lockwood, M., Stamper, R., \& Wild, M. N. 1999, Nature, 399, 437

Mendoza, B. 1997, ApJ, 483, 523

Ortiz, A., Solanki, S. K., Domingo, V., Fligge, M., \& Sanahuja, B. 2002, A\&A, 388,1036

Parker, E. N. 1987, ApJ, 312, 868

Parker, E. N. 1995, ApJ, 440, 415

Preminger, D. G., \& Walton, S. R. 2005, Geophys. Res. Lett., 32, L14109

Reid, G. C. 1987, Nature, 329, 142

Rind, D. 2002, Science, 296, 673

Schrijver, C. J., \& Harvey, K. L. 1994, Sol. Phys., 150, 1

Shindell, D. T. 2001, Geophys. Res. Lett., 28, 1551

Sinnhuber, B.-M., von der Gathen, P., Sinnhuber, M., et al. 2005, Atm. Chem. \& Phys. Disc., 5, 12103

Solanki, S. K. 2003, A\&AR, 11, 153

Solanki, S. K., \& Fligge, M. 1998, Geophys. Res. Lett., 25, 341

Solanki, S. K., \& Fligge, M. 1999, Geophys. Res. Lett., 26, 2465

Solanki, S. K., \& Krivova, N. A. 2004, Sol. Phys., 224, 197

Solanki, S. K., Schüssler, M., \& Fligge, M. 2000, Nature, 408, 445

Solanki, S. K., Schüssler, M., \& Fligge, M. 2002, A\&A, 383, 706

Solanki, S. K., Krivova, N. A., \& Wenzler, T. 2005, Adv. Sp. Res., 35, 376

Stott, P. A., Jones, G. S., \& Mitchell, J. F. B. 2003, J. Clim., 16, 4079

Unruh, Y. C., Solanki, S. K., \& Fligge, M. 1999, A\&A, 345, 635

Waldmeier, M. 1961, The sunspot-activity in the years 1610-1960 (Zürich: Schulthess)

Wang, Y.-M., Lean, J. L., \& Sheeley, N. R. 2005, ApJ, 625, 522

Wenzler, T. 2005, Ph.D. Thesis, ETH Zürich

Wenzler, T., Solanki, S. K., Krivova, N. A., \& Fluri, D. M. 2004, A\&A, 427, 1031

Wenzler, T., Solanki, S. K., \& Krivova, N. A. 2005, A\&A, 432, 1057

Wenzler, T., Solanki, S. K., Krivova, N. A., \& Fröhlich, C. 2006, A\&A, 460, 583

White, O. R., Skumanich, A., Lean, J., Livingston, W. C., \& Keil, S. L. 1992,

Publ. Astron. Soc. Pac., 104, 1139

Willson, R. C. 1997, Science, 277, 1963

Willson, R. C., \& Hudson, H. S. 1988, Nature, 332, 810

Willson, R. C., \& Hudson, H. S. 1991, Nature, 351, 42

Woods, T. N., Prinz, D. K., Rottman, G. J., \& et al. 1996, J. Geophys. Res., 101 (D6), 9541

Wright, J. T. 2004, AJ, 128, 1273

Zhang, Q., Soon, W. H., Baliunas, S. L., et al. 1994, ApJ, 427, L111 